\title{
Optical design of an in vivo laparoscopic lighting system
}

\author{
Xiaolong Liu \\ Reza Yazdanpanah Abdolmalaki \\ Gregory J. Mancini \\ Jindong Tan
}




\title{
Optical design of an in vivo laparoscopic lighting system
}

\author{
Xiaolong Liu, ${ }^{\mathrm{a}, \star}$ Reza Yazdanpanah Abdolmalaki, ${ }^{a}$ Gregory J. Mancini, ${ }^{\mathrm{b}}$ and Jindong Tan ${ }^{\mathrm{a}}$ \\ aniversity of Tennessee, Department of Mechanical, Aerospace, and Biomedical Engineering, Knoxville, Tennessee, United States \\ bUniversity of Tennessee Medical Center, Department of Surgery, Knoxville, Tennessee, United States
}

\begin{abstract}
This paper proposes an in vivo laparoscopic lighting system design to address the illumination issues, namely poor lighting uniformity and low optical efficiency, existing in the state-of-the-art in vivo laparoscopic cameras. The transformable design of the laparoscopic lighting system is capable of carrying purposefully designed freeform optical lenses for achieving lighting performance with high illuminance uniformity and high optical efficiency in a desired target region. To design freeform optical lenses for extended light sources such as LEDs with Lambertian light intensity distributions, we present an effective and complete freeform optical design method. The procedures include (1) ray map computation by numerically solving a standard MongeAmpere equation; (2) initial freeform optical surface construction by using Snell's law and a lens volume restriction; (3) correction of surface normal vectors due to accumulated errors from the initially constructed surfaces; and (4) feedback modification of the solution to deal with degraded illuminance uniformity caused by the extended sizes of the LEDs. We employed an optical design software package to evaluate the performance of our laparoscopic lighting system design. The simulation results show that our design achieves greater than $95 \%$ illuminance uniformity and greater than $89 \%$ optical efficiency (considering Fresnel losses) for illuminating the target surgical region. ๑ 2017 Society of Photo-Optical Instrumentation Engineers (SPIE) [DOI: 10.1117/1.JBO.22.12.125003]
\end{abstract}

Keywords: laparoscopic lighting; freeform optical design; medical device.

Paper 170308RR received May 16, 2017; accepted for publication Nov. 17, 2017; published online Dec. 9, 2017.

\section{Introduction}

The development of in vivo laparoscopic cameras, which can be inserted in the abdominal cavity through a small incision and provide real-time visual feedback for surgeons, has been an important on-going research topic to improve medical performance for minimally invasive surgery (MIS). Compared with conventional rigid long-stick laparoscopic video systems, the benefits of employing in vivo laparoscopic cameras include: (1) better triangulation capabilities and wider field of view (FOV); ${ }^{1,2}$ (2) less internal/external collisions with other surgical instruments; $;^{3-5}$ (3) unrestricted intra-abdominal manipulation and more intuitive control, ${ }^{6-8}$ and (4) no need of a dedicated port that reduces abdomen tissue damages. ${ }^{9}$

Although in vivo laparoscopic cameras feature the advantages mentioned above, they are still in their infancy for real MIS tasks. One of the major issues that impedes the in vivo cameras from being practical is their inferior imaging performance due to three main reasons. First, the sizes of imaging sensors and optical lenses applied in in vivo cameras are limited by the compact dimensions of the cameras. It is a great challenge to achieve imaging resolutions comparable to the off-the-shelf conventional laparoscopic cameras, such as Stryker HD 3-Chip series cameras, ${ }^{10}$ the Olympus $4 \mathrm{~K}$ Camera, ${ }^{11}$ etc. Second, lighting systems play a crucial role in determining the quality of surgical images beyond the imaging sensors themselves. The state-ofthe-art in vivo laparoscopic cameras employ bare LEDs or LEDs combined with poorly designed reflectors. The uncontrolled light beams therefore either waste most of their energy to illuminate areas outside the camera's $\mathrm{FOV}^{6}$ or result in bright center and dark margins in the plane of an imaging sensor. ${ }^{2,5}$ In contrast, the conventional laparoscopic video systems introduce external xenon/LED light sources into abdominal cavities via fiber-optics inside the rods. Illumination optical designs are usually only applied to the rods to improve energy efficiency and illuminance uniformity. ${ }^{12}$ Last, due to the commercialization of conventional laparoscopic video systems, image processing software is well developed with sophisticated image enhancement techniques, which further increase the gap between in vivo laparoscopic cameras and conventional laparoscopic video systems.

The objective of this paper is to propose a solution for addressing the illumination issues existing in the state-of-theart in vivo laparoscopic cameras. We aim at pushing in vivo laparoscopic cameras to take one step forward toward practical use in MIS. There are two major challenges for achieving this goal. The first challenge is the deployment of light sources and nonimaging optical lenses in an in vivo laparoscopic camera, whose outer diameter is limited by a trocar's inner diameter (3 to $30 \mathrm{~mm}^{13}$ ). Considering the common deployment of LEDs that surrounds an imaging sensor, it will be difficult to install an additional optical system for the LEDs in such a small area for uniform illumination. In addition, the coaxial configuration of an imaging sensor and light sources results in the lack of shadow depth cues in output two-dimensional (2-D) images. In some cases, shadow depth cues are desired by surgeons to compensate for degraded visual information and improve surgical performance. ${ }^{14,15}$ The second challenge is the design of nonimaging optics for the light sources. The lighting system should 
satisfy the following requirements: (1) uniform illuminance distribution on a target surgical area; (2) high optical efficiency, which means maximally projecting light rays only inside a camera's FOV; and (3) compact design to fit in the limited space of an in vivo laparoscopic camera.

In this paper, we propose a transformable design of an in vivo laparoscopic camera system that is able to carry well-designed freeform optical lenses. We also develop an effective freeform optical lens design method for the LEDs to achieve desired illumination on target surgical areas. As conceptually shown in Fig. 1, the device is delivered into the abdominal cavity with the folded mode, as shown in Fig. 1(b). The device transformation is activated to expose the lighting system and the imaging system in the abdominal cavity after the device is magnetically anchored inside the abdominal wall, as shown in Fig. 1(c). It is a crucial task to design freeform optical lenses that can be harbored on the wings [Fig. 1(a)-8] and can meet the lighting requirements discussed previously. To generate a smooth freeform optical surface, the integrability condition of optical surface normal vectors ${ }^{16}$ needs to be enforced. Although the nonstandard Monge-Ampere equation, which governs the freeform lens design problem, ${ }^{17}$ can guarantee such integrability condition, it is very difficult to compute a convergent solution. Instead, we propose an efficient ray-mapping-based method to generate a smooth freeform surface for the lighting system. A ray map between the light source and the target area can be formulated in an $L^{2}$ Monge-Kantorovich problem and governed by a standard Monge-Ampere equation. We introduce an effective numerical method to solve the standard Monge-Ampere equation. This method employs a sequence of higher order quasilinear PDEs to approximate the solution of the lower order nonlinear PDEs (the standard Monge-Ampere equation). ${ }^{18,19}$ Based on a computed ray map, an initial optical surface can be constructed by Snell's law. ${ }^{20}$ This initial surface construction method suffers accumulated errors on the surface's normal vectors. For improving the optical design performance, we propose an iterative optimization technique to correct the initial surface. To reduce the distortion from extended sizes of LEDs, we employ a feedback modification method to improve illuminance uniformity. Our proposed freeform optical design method features an easy-to-implement numerical solver, fast convergent

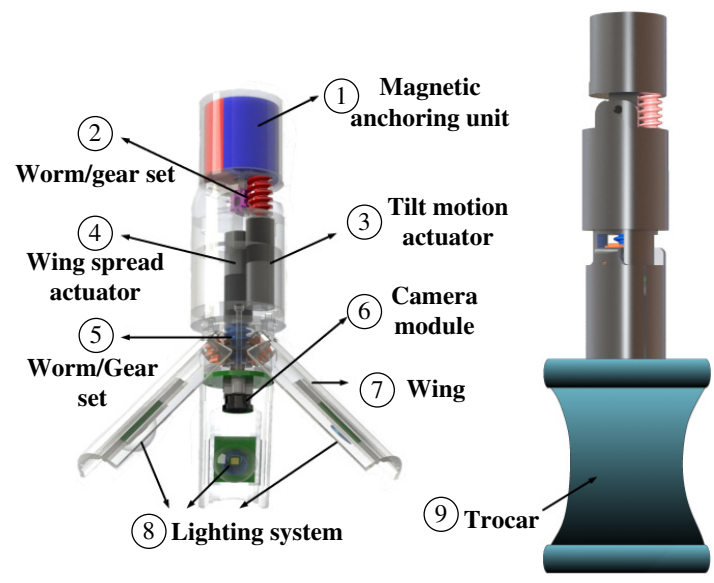

(a) speed, and optimized optical performance that have been verified using an optical design software package. This method is not only very effective to design the lighting system for the in vivo laparoscopic camera but it also can serve as a general purpose freeform surface design method for other related applications.

\section{Method}

In this section, we first briefly describe our in vivo robotic laparoscopic system design, its application scenario, and discuss the design requirements of the lighting system. Then, we elaborate our proposed freeform optical design method to achieve the lighting requirements.

\subsection{Configuration of In Vivo Laparoscopic System}

Figure 1 shows the configuration of the in vivo laparoscopic system for MIS, which consists of a camera module (a)-(6), a lighting system (a)-8, actuation mechanisms for the robot's transformation (a)-(4)(5, and orientation control (a)-(2)(3), and a magnetic anchoring unit (a)-(1) paired with an external magnetic holder for affixing the robot against the inner side of an abdominal wall.

The outer diameter of the folded mode [Fig. 1(b)] is designed as $17 \mathrm{~mm}$, which can fit in a trocar with $20 \mathrm{~mm}$ sleeve diameter. ${ }^{21}$ An external magnetic holder navigates the inserted robotic platform to a desired location and anchors it inside the abdominal wall, as shown in Fig. 1(c). Then, the wings [Fig. 1(a)-(7)] are extended to expose the camera module and the lighting system inside the abdominal cavity for visualizing the surgical area. Imaging data are transmitted to display on a monitor screen for guiding surgical tasks.

\subsection{Design Requirements of Lighting System}

To help with clearly discussing the design requirements of the lighting system, we use the CMOS imaging sensor OV7850 22 and a pin-hole lens ${ }^{23}$ with FOV $48 \mathrm{deg}$ in horizontal and $36 \mathrm{deg}$ in vertical as a benchmark setup. The selected imaging sensor is with a sensitivity of $16 \mathrm{~V} /$ lux-s, an imaging array size of $752 \times 548$, and a pixel size of $6.0 \mu \mathrm{m} \times 6.0 \mu \mathrm{m}$. To enable

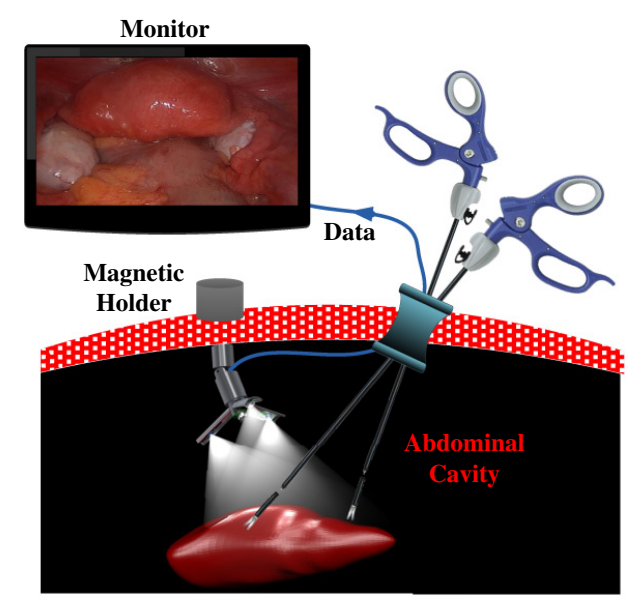

(c)

Fig. 1 Conceptual illustration of the transformable in vivo robotic laparoscopic lighting system. (a) The extended mode of the lighting system; (b) the folded mode; and (c) an application scenario of the lighting system. 
Table 1 Specifications of lighting requirements.

\begin{tabular}{lcc} 
Characteristic & Value & Units \\
\hline Illuminance (at $100 \mathrm{~mm}$ ) & 10,000 & $\mathrm{Ix}$ \\
Illumination radius (at $100 \mathrm{~mm}$ ) & 80 & $\mathrm{~mm}$ \\
Total luminous flux & 200.96 & $\mathrm{Im}$ \\
Illumination distance & 50 to 100 & $\mathrm{~mm}$ \\
\hline
\end{tabular}

a comparable camera system in proper working condition, a maximum illuminance of $4000 \mathrm{~lx}$ on the target illuminated area is operated at a distance of $50 \mathrm{~mm} .^{6}$ The number of light rays entering the imaging sensor heavily depends on the form of an illuminated area and is difficult to characterize and control. To ensure sufficient light that enters the imaging sensor, we conservatively require the minimum illuminance to be $10,000 \mathrm{~lx}$ at a distance of $100 \mathrm{~mm}$. The illumination radius is set as $80 \mathrm{~mm}$ to contain the camera's FOV when the camerato-target distance is $100 \mathrm{~mm}$. To uniformly achieve the illuminance of 10,000 lx within the area, the minimum total luminous flux of the lighting system is calculated to be $200.96 \mathrm{~lm}$. The lighting requirements are summarized in Table 1.

\subsection{Problem Formulation of Optical Lens Design for Lighting System}

Benefiting from the state-of-the-art high efficiency tiny LEDs, e.g., Cree Xlamp XQ-E $(1.6 \mathrm{~mm} \times 1.6 \mathrm{~mm} \times 0.8 \mathrm{~mm}$, $128 \mathrm{~lm}$ at $2.9 \mathrm{~V}$ and $350 \mathrm{~mA}$ ), Nichia NCSWE17A $(1.7 \mathrm{~mm} \times 1.7 \mathrm{~mm} \times 0.3 \mathrm{~mm}, 118 \mathrm{~lm}$ at $3.0 \mathrm{~V}$ and $350 \mathrm{~mA})$, only one or two of such LEDs can satisfy the luminous flux requirements in Table 1.

Optical lenses are considered to have better performance on light beam control than reflectors. ${ }^{24}$ Our proposed in vivo laparoscopic lighting system uniquely provides the feasibility to integrate optical lens with the LEDs for highly efficient, uniform illumination on target surgical areas. Figure 2 conceptually shows the configuration of the lighting system. Three LEDs are separately installed on three wings to compensate for energy

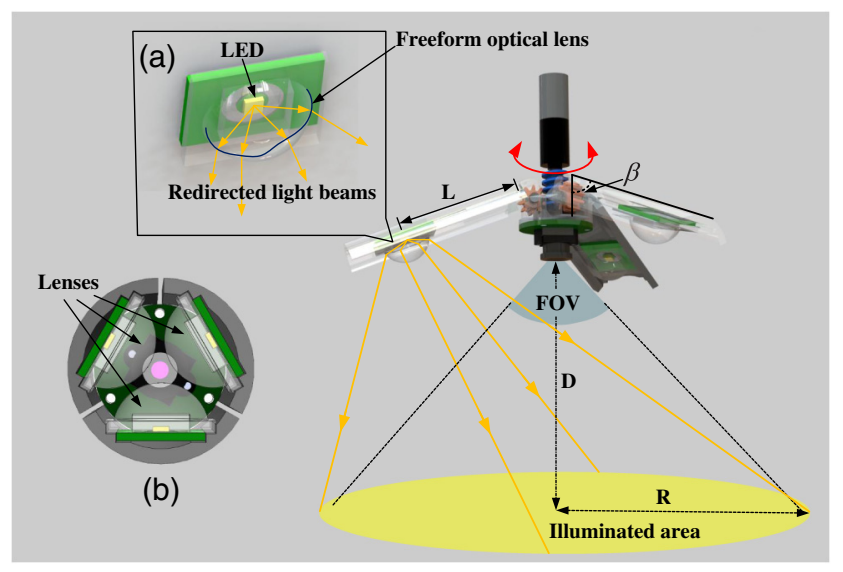

Fig. 2 Configuration of the lighting system. (a) The light beams are redirected by using a freeform optical lens. (b) The bottom view of the folded mode. loss in the lighting system. One actuator drives the wings to reach a common opening angle $\beta$. For each LED, all the light rays should be redirected and uniformly distributed on the illuminated area by applying a freeform optical lens, as shown in Fig. 2(a). In addition, all the freeform lenses should be able to fit in the folded mode, as shown in Fig. 2(b).

Therefore, the key problem of this research can be recognized as how to design a proper freeform optical lens for each LED to redirect the light beams to be uniformly distributed on a target surgical area. In this paper, we contribute an effective method to solve this problem, which is detailed in the following sections.

\subsection{Related Work of Freeform Optical Lens Design}

The freeform optical design problem is usually approached under the assumption of using a zero-étendue source (point source) and governed by a nonstandard Monge-Ampere equation, ${ }^{17,16,25,26}$ which is a second-order nonlinear PDE. However, due to the high nonlinearity of the nonstandard Monge-Ampere equation, it is very difficult to find an effective and easy-toimplement numerical method to compute a convergent solution.

Alternative methods are developed to avoid solving the nonstandard Monge-Ampere equation directly, such as the supporting quadratics methods. ${ }^{27-29}$ However, as the number of quadratics grows, the computational cost goes up quickly. The ray-mapping method is another effective way to design freeform lenses, ${ }^{20,30}$ which basically follows two steps: (1) ray map generation and (2) optical surface construction. The main challenge in the ray-mapping method is to enforce integrability conditions on normal vectors of constructed optical surfaces. This ray-mapping requirement can be formulated in an $L^{2}$ MongeKantorovich problem, which can be equivalently represented by a standard Monge-Ampere equation. ${ }^{31}$ The ray-mapping computation methods, however, are either hard to numerically implement $^{32,33}$ or tricky to get a convergent solution. ${ }^{31,34}$ In this paper, we contribute an effective ray-mapping method for freeform lens design. This method features fast convergence speed and easy numerical implementation. Based on the computed ray map, a freeform optical surface is constructed by the following procedures, which include an initial freeform optical surface construction, correction of normal vectors on the freeform surface, and feedback modification of desired target region illuminance distribution. The detailed design method is elaborated in the following sections.

\subsection{Design Framework of Freeform Optical Lens}

Figure 3 shows the framework of our proposed freeform optical lens design method for the in vivo laparoscopic lighting system. The design requirements, the LED's luminous intensity distribution $I_{s}(\theta, \phi)$, and the desired illuminance distribution $E_{t}(x, y)$ on a target region are initialized as the design inputs. The raymapping method proposed in Sec. 2.6 requires both the LED and the illuminated area to be represented in the forms of illuminance distributions. The conversion of representing the LED from luminous intensity distribution $I_{s}(\theta, \phi)$ to illuminance distribution $E_{s}(\xi, \eta)$ is detailed in Sec. 2.7. According to the computed ray map, an initial freeform optical surface is constructed in Sec. 2.8.1. To reduce accumulated errors of normal vectors on the freeform surface, a freeform surface correction method is proposed in Sec. 2.8.2. Due to the fact that the LEDs are extended light sources, the solution derived in the initial design 


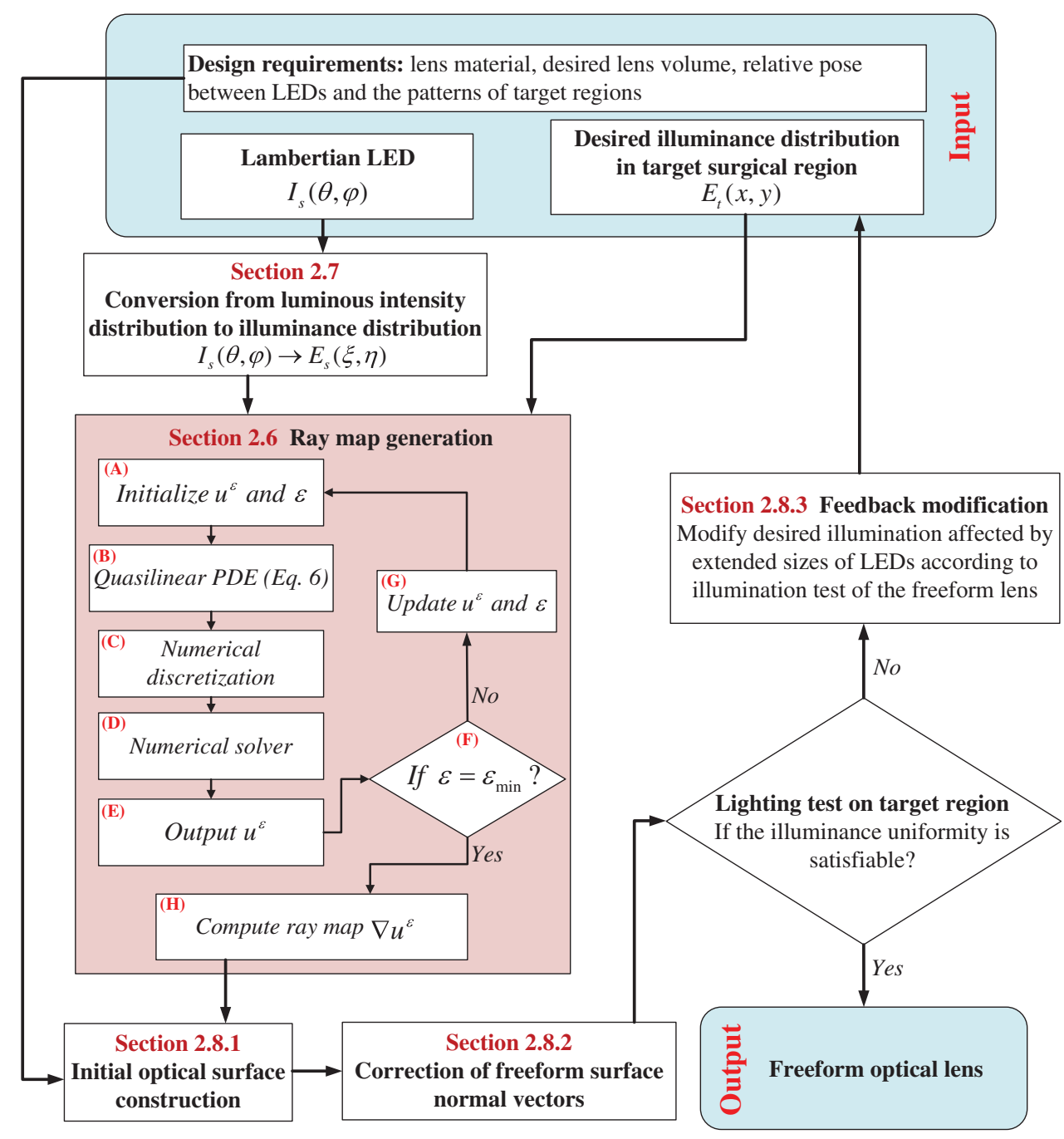

Fig. 3 Framework of freeform optical lens design.

will be degraded since a point light source is assumed in the design of freeform optical lenses. A feedback modification procedure introduced in Sec. 2.8.3 is employed to address this issue. A few iterations of the feedback modification are required to generate the final design of the freeform optical lens.

\subsection{Ray-Mapping Method}

Let $E_{s}(\xi, \eta)$ and $E_{t}(x, y)$ represent the LED's illuminance distribution and the prescribed target illuminance distribution, respectively. As shown in Fig. 4, our objective is to find the ray-mapping function $\hat{\mathbf{x}}=\phi(\zeta)$ that transfers $E_{s}$ to $E_{t}$, where $\zeta=(\xi, \eta)$ and $\hat{\mathbf{x}}=(x, y)$ are Cartesian coordinates confined in the source domain $\Omega_{s}$ and the target domain $\Omega_{t}$. The above statements are recognized as a special case of the $L^{2}$ Monge-Kantorovich problem. Under the assumption that there is no energy lost in transport, $\phi$ should satisfy

$\int_{\Omega_{t}} E_{t}(\hat{\mathbf{x}}) \mathrm{d} \hat{\mathbf{x}}=\int_{\Omega_{s}} E_{s}(\zeta) \mathrm{d} \zeta$.

According to the mapping $\hat{\mathbf{x}}=\phi(\zeta)$, Eq. (1) can be represented as
$E_{t}[\phi(\zeta)] \operatorname{det}[\nabla \phi(\zeta)]=E_{s}(\zeta)$

Brenier's theorem ${ }^{35}$ states that there exists a unique solution $\phi=\tilde{\phi}$ to an $L^{2}$ Monge-Kantorovich problem, which can be characterized as the gradient of a convex potential $\tilde{\phi}=\nabla u$. Substitute $\nabla u$ in Eq. (2), then $u$ is a solution of the standard Monge-Ampere equation

$$
E_{t}[\nabla u(\zeta)] \operatorname{det} \nabla^{2} u(\zeta)=E_{s}(\zeta)
$$

\subsubsection{Method for solving standard Monge-Ampere equation}

It is observed that a weak solution of a lower order nonlinear PDE can be approximated by a sequence of higher order quasilinear PDEs. ${ }^{18}$ To approximate the solution of a standard Monge-Ampere equation, which is a second-order nonlinear PDE, a biharmonic operator with fourth-order partial derivatives is a good option. ${ }^{19}$

The approximated solution $u^{\epsilon}$ of Eq. (3) can thus be computed from

$-\epsilon \Delta^{2} u^{\epsilon}+E_{t}\left[\nabla u^{\epsilon}(\zeta)\right] \operatorname{det} \nabla^{2} u^{\epsilon}(\zeta)-E_{s}(\zeta)=0$, 


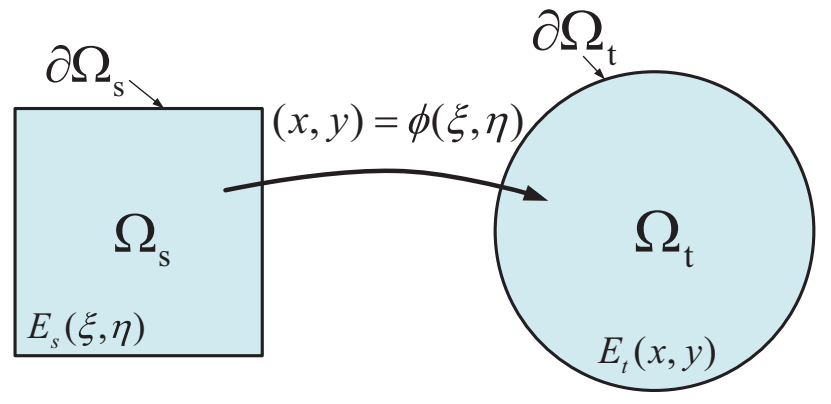

Fig. 4 Energy transportation from the light source domain $\Omega_{s}$ to the target domain $\Omega_{t}$.

where $\epsilon>0$ and $\lim _{\epsilon \rightarrow 0^{+}} u^{\epsilon}$ is a moment solution if the limit exists. The inner points of $\Omega_{s}$ should satisfy Eq. (4). The points on the boundary $\partial \Omega_{s}$ of $\Omega_{s}$ should be mapped to the boundary $\partial \Omega_{t}$ of $\Omega_{t}$. According to $\hat{\mathbf{x}}=\nabla u^{\epsilon}(\zeta)$, a Neumann boundary condition $(\mathrm{BC})$ can be formulated as

$f\left[\nabla u^{\epsilon}(\zeta)\right]=0$,

where $f$ is the mathematical representation of the shape of $\partial \Omega_{t}$. In combining Eqs. (4) and (5), the ray map can be computed from the following quasilinear PDE and the Neumann BC:

$$
\left\{\begin{array}{ll}
-\epsilon \Delta^{2} u^{\epsilon}+E_{t}\left[\nabla u^{\epsilon}(\zeta)\right] \operatorname{det} \nabla^{2} u^{\epsilon}(\zeta)-E_{s}(\zeta)=0, & \zeta \in \Omega_{s} \\
\mathrm{BC}: f\left[\nabla u^{\epsilon}(\zeta)\right]=0, & \zeta \in \partial \Omega_{s}
\end{array} .\right.
$$

$\left\{\begin{array}{l}-\epsilon \Delta^{2} u^{\epsilon}+E_{t}\left(\frac{\partial u^{e}}{\partial \xi}, \frac{\partial u^{e}}{\partial \eta}\right)\left[\frac{\partial^{2} u^{e}}{\partial \xi^{2}} \frac{\partial^{2} u^{e}}{\partial \eta^{2}}-\left(\frac{\partial^{2} u^{e}}{\partial \xi \partial \eta}\right)^{2}\right]-E_{s}(\xi, \eta)=0 \\ \mathrm{BC}: f\left(\frac{\partial u^{e}}{\partial \xi}, \frac{\partial u^{e}}{\partial \eta}\right)=0\end{array}\right.$

The discretization of first-order and second-order partial derivatives in Eq. (8) adapts the central finite difference method on the inner region of $\Omega_{s}$, and the forward/backward finite difference method on the boundary region $\partial \Omega_{s}$ with secondorder truncation errors. The discretization of the biharmonic term $\Delta^{2} u^{\epsilon}$ in Eq. (8) can be achieved by a 13-point stencil ${ }^{37}$

$$
\begin{aligned}
\Delta^{2} u^{\epsilon}= & \frac{1}{h^{4}}\left[20 u_{i, j}^{\epsilon}-8\left(u_{i+1, j}^{\epsilon}+u_{i-1, j}^{\epsilon}+u_{i, j+1}^{\epsilon}+u_{i, j-1}^{\epsilon}\right)\right. \\
& +2\left(u_{i+1, j+1}^{\epsilon}+u_{i-1, j+1}^{\epsilon}+u_{i-1, j-1}^{\epsilon}+u_{i+1, j-1}^{\epsilon}\right) \\
& \left.+\left(u_{i+2, j}^{\epsilon}+u_{i-2, j}^{\epsilon}+u_{i, j+2}^{\epsilon}+u_{i, j-2}^{\epsilon}\right)\right]
\end{aligned}
$$

where we represent $\left(\xi_{i}, \eta_{j}\right)$ as $(i, j)$ for short. However, undefined points are introduced when the near-boundary points are discretized by using the 13-point stencil in Eq. (9). Figure 5 demonstrates an example that the center of a 13point stencil $u_{i, j}^{\epsilon}$ (marked by the red dot) locates in the nearboundary region. In this case, $u_{i-2, j}^{\epsilon}$ and $u_{i, j-2}^{\epsilon}$ (marked by the gray dots) are outside the source region $\Omega_{s}$. The approximations of undefined $u_{i+2, j}^{\epsilon}, u_{i-2, j}^{\epsilon}, u_{i, j+2}^{\epsilon}, u_{i, j-2}^{\epsilon}$ are required, which can be computed using the following formulas:

\subsubsection{Numerical technique for computing ray map}

The ray map $\nabla u^{\epsilon}$, which is governed by Eq. (6), is computed by the procedures shown in Fig. 3 "ray map generation." The main idea of the proposed numerical technique is to iterate the approximated $u^{\epsilon}$ by updating $\epsilon$. To be specific, $\epsilon$ is set as a sequence of gradually reduced constant values, e.g., $1,10^{-1}$, $10^{-2}$, and so on. In each iteration, an initial guess $u^{\epsilon}$ is first provided either by the output $u^{\epsilon}$ of the last iteration or manually selected (in the first iteration). The number of iterations depends on the number of $\epsilon$ in the sequence. We can start the iteration with $\epsilon=1$ to approximate the solution $u^{\epsilon}$ of Eq. (3). When $\epsilon$ approaches $0^{+}$, Eq. (4) is equivalent to Eq. (3). But it does not mean that the best approximated solution $u^{\epsilon}$ can be found when we finalize $\epsilon$ as 0 in the iteration procedure.

The error of $\left\|u-u_{h}^{\epsilon}\right\|$ is bounded by

$\left\|u-u_{h}^{\epsilon}\right\| \leq\left\|u-u^{\epsilon}\right\|+\left\|u^{\epsilon}-u_{h}^{\epsilon}\right\|$,

where $u_{h}^{\epsilon}$ denotes the numerical solution of Eq. (6) with a mesh size $h$. The finalized value of $\epsilon$ in Eq. (6) is related to $h$ for achieving optimized convergent speed and minimum error. This relationship depends on the norm to be used. According to the numerical experiments analyzed in Ref. 36, the minimum global error can be achieved when $\epsilon=h,\left\|u-u_{h}^{\epsilon}\right\|_{H^{1}}$.

To numerically discretize Eq. (6), the quasilinear PDE and the $\mathrm{BC}$ are reformulated as

$(\xi, \eta) \in \Omega_{s}$

$(\xi, \eta) \in \partial \Omega_{s}$

$$
\left\{\begin{array}{c}
u_{i-2, j}^{\epsilon}=u_{i, j}^{\epsilon}-2 h\left(\frac{\partial u^{e}}{\partial \xi}\right)_{i-1, j}+O\left(h^{2}\right), \\
u_{i, j-2}^{\epsilon}=u_{i, j}^{\epsilon}-2 h\left(\frac{\partial u^{e}}{\partial \eta}\right)_{i, j-1}+O\left(h^{2}\right), \\
u_{i+2, j}^{\epsilon}=u_{i, j}^{\epsilon}+2 h\left(\frac{\partial u^{\epsilon}}{\partial \xi}\right)_{i+1, j}+O\left(h^{2}\right), \\
u_{i, j+2}^{\epsilon}=u_{i, j}^{\epsilon}+2 h\left(\frac{\partial u^{\epsilon}}{\partial \eta}\right)_{i, j+1}+O\left(h^{2}\right),
\end{array}\right.
$$

where $u_{i, j}^{\epsilon}$ denotes a near-boundary value in the grid of $\Omega_{s} ; h$ is the mesh size in both $\xi$ and $\eta$ directions; $\left(\partial u^{\epsilon} / \partial \xi\right)_{i-1, j}$,

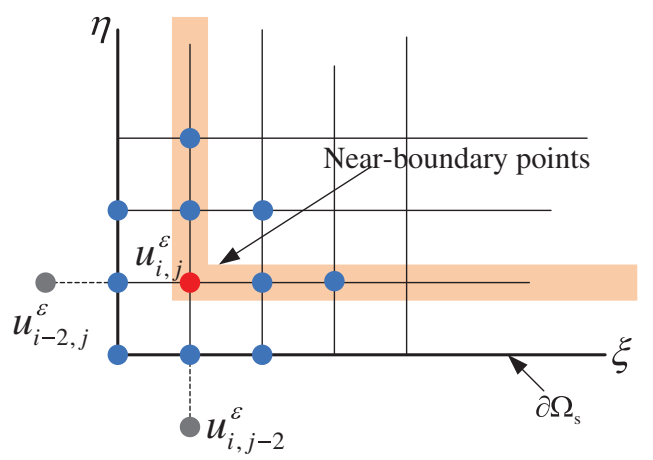

Fig. 5 Discretization of the biharmonic term at the near-boundary region. 
$\left(\partial u^{\epsilon} / \partial \eta\right)_{i, j-1},\left(\partial u^{\epsilon} / \partial \xi\right)_{i+1, j},\left(\partial u^{\epsilon} / \partial \eta\right)_{i, j+1}$ are first-order partial derivatives on $\partial \Omega_{s}$, which can be determined by the BC in Eq. (8).

The numerical discretization of Eq. (8) results in a set of nonlinear equations that can be represented in the form of

$\mathbf{F}\left(\mathbf{U}^{\epsilon}\right)=0$,

where $\mathbf{U}^{\epsilon}$ denotes a vector of variables $u^{\epsilon}$. The Newton's method is chosen as the numerical solver [Fig. 3(d)] to compute $u^{\epsilon}$ [Fig. 3(e)]. Figure 3(f) compares the $\epsilon$ in the current iteration with $\epsilon_{\min }=h$. If $\epsilon>\epsilon_{\min }$, the initial $u^{\epsilon}$ and $\epsilon$ in Fig. 3(a) will be updated with the computed $\mathbf{U}^{\epsilon}$ and a decreased $\epsilon$. Otherwise, the final ray map [Fig. 3(h)] will be computed using the gradient of the numerical solution $\mathbf{U}^{\epsilon}$ from the current iteration.

\subsection{Luminous Intensity to Illuminance Conversion}

The ray-mapping technique proposed in the previous section requires to use the illuminance distribution $E_{s}$ of the LED. However, the LED employed in this work is a Lambertian light source, which is usually described by a luminous intensity distribution $I=I_{0} \cos \theta$ (unit: $\mathrm{lm} \cdot \mathrm{sr}^{-1}$ ) in a hemispherical space. $\theta$ denotes the polar angle of light ray, and $I_{0}$ represents the luminous intensity at $\theta=0 \mathrm{deg}$. We apply the stereographic projection method ${ }^{17}$ to convert the source's luminous intensity to the illuminance distribution, which is defined on a plane. The main idea of this method is to project the light energy with an emitting direction $\mathbf{S P}=\left(x_{u}, y_{u}, z_{u}\right)$ onto the $\xi-\eta$ plane at coordinates $\zeta=(\xi, \eta)$, as shown in Fig. 6. The final form of the illuminance distribution $E_{s}(\xi, \eta)$ on the $\xi-\eta$ plane is represented as

$E_{s}(\xi, \eta)=\frac{4 I_{0}\left(1-\xi^{2}-\eta^{2}\right)}{\left(1+\xi^{2}+\eta^{2}\right)^{3}}$,

where $\xi^{2}+\eta^{2} \leq 1$. For the grid points $\xi^{2}+\eta^{2} \geq 1$, we define $E_{s}(\xi, \eta)=0$.

\subsection{Freeform Optical Surface Construction}

\subsubsection{Initial surface construction}

Based on the computed ray map, each pair of coordinates $\left(\xi_{i}, \eta_{j}\right)$ in $\Sigma_{L}\left\{x_{L}, y_{L}, z_{L}\right\}$ on the source grid can be mapped to a point $\mathbf{T}_{i, j}^{\prime}=\left[x_{i}^{\prime}, y_{j}^{\prime}, z^{\prime}\left(x_{i}, y_{j}\right)\right]$ in $\Sigma_{G}\left\{x_{G}, y_{G}, z_{G}\right\}$ on the target plane, where $i$ and $j$ represent discretization indices of the light source. According to the rotation matrix $\mathbf{R}$ and the translational vector

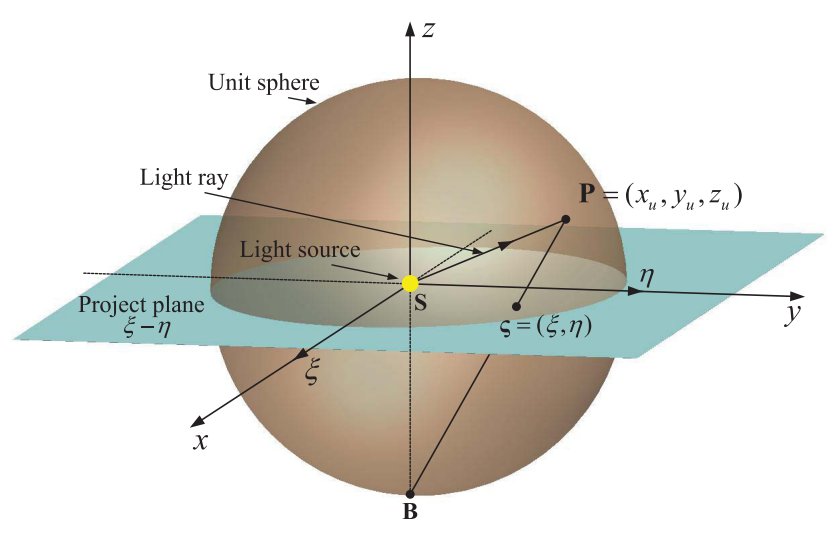

Fig. 6 Illustration of stereographic projection.
T between $\Sigma_{G}$ and $\Sigma_{L}, \mathbf{T}_{i, j}^{\prime}$ can be represented as $\mathbf{T}_{i, j}$ in $\Sigma_{L}$, as shown in Fig. 7(b). A unit incident ray vector from the light source is defined by $\mathbf{I}_{i, j}=\left(x_{u_{i, j}}, y_{u_{i, j}}, z_{u_{i, j}}\right)$, where $x_{u_{i, j}}, y_{u_{i, j}}$, and $z_{u_{i, j}}$ are functions of $\left(\xi_{i}, \eta_{j}\right)$. We employ an easy-to-implement surface construction method ${ }^{20}$ to design an initial optical surface for the light source. The main idea of this method is to first construct one curve with a sequence of points $\mathbf{p}_{1,1}, \ldots, \mathbf{p}_{1, n}$, as shown in Fig. 7(a)-1. Then, the generated curve is used to compute the surface points along the direction in Fig. 7(a)-(2).

As shown in Fig. 7(a), we define $\mathbf{O}_{i, j}$ as a unit out-going ray from the optical surface, and formulate it as

$\mathbf{O}_{i, j}=\frac{\mathbf{T}_{i, j}-\mathbf{p}_{i, j}}{\left|\mathbf{T}_{i, j}-\mathbf{p}_{i, j}\right|}$,

where $\mathbf{p}_{i, j}$ denotes a point to be constructed on the surface. In Fig. 7(a)-(1), considering the desired lens dimensions, an initial point $\mathbf{p}_{1,1}$ can be manually selected according to a desired lens volume. Thus, $\mathbf{O}_{1,1}$ is calculated with Eq. (13). The normal vector at $\mathbf{p}_{i, j}$ can be computed by Snell's law

$\mathbf{N}_{i, j}=\frac{n_{0} \mathbf{O}_{i, j}-n_{1} \mathbf{I}_{i, j}}{\left|n_{0} \mathbf{O}_{i, j}-n_{1} \mathbf{I}_{i, j}\right|}$,

where $n_{0}$ denotes the refractive index of the medium surrounding the lens, and $n_{1}$ represents the refractive index of the lens. The coordinates of the next point $\mathbf{p}_{1,2}$ on the curve is computed by solving the intersection point between the light ray $\mathbf{I}_{1,2}$ and the plane defined by $\mathbf{p}_{1,1}$ and $\mathbf{N}_{1,1}$. The curves in direction (2) can be computed using the points on the first curve as initial points.

\subsubsection{Correction of surface normal vectors}

Although this method provides an easy way to construct the freeform surface with required lens dimensions, it cannot guarantee that the computed normal vector $\mathbf{N}_{i, j}$ at $\mathbf{p}_{i, j}$ are perpendicular to the vectors between $\mathbf{p}_{i, j}$ and its adjacent points $\mathbf{p}_{i+1, j}, \mathbf{p}_{i, j+1}$, as shown in Fig. 7(b) due to accumulated errors. To address this problem and improve the illumination performance, we introduce an iterative optimization technique to correct the constructed initial surface for better fitting the normal vectors. ${ }^{38}$ Ideally, if the surface mesh is fine enough, a surface point $\mathbf{p}_{i, j}$ and the normal vector $\mathbf{N}_{i, j}$ at this point should satisfy the following constraints:

$\left(\mathbf{p}_{i+1, j}-\mathbf{p}_{i, j}\right) \cdot \mathbf{N}_{i, j}=0$,

$\left(\mathbf{p}_{i, j+1}-\mathbf{p}_{i, j}\right) \cdot \mathbf{N}_{i, j}=0$.

Assume the optical surface is constructed by $N$ points. By substituting $\mathbf{p}_{i, j}$ with $\rho_{i, j} \mathbf{I}_{i, j}$ in Eqs. (15) and (16), we have $N$ constraints $F_{1}, \ldots, F_{N}$

$$
\begin{aligned}
F_{k}(\boldsymbol{\rho})= & \|\left(\rho_{i+1, j} \mathbf{I}_{i+1, j}-\rho_{i, j} \mathbf{I}_{i, j}\right) \\
& \cdot \mathbf{N}_{i, j}\|+\|\left(\rho_{i, j+1} \mathbf{I}_{i, j+1}-\rho_{i, j} \mathbf{I}_{i, j}\right) \cdot \mathbf{N}_{i, j} \|=0,
\end{aligned}
$$

where $k=1, \ldots, N, \rho_{i, j}$ denotes the distance between $\mathbf{S}$ and the surface point $\mathbf{p}_{i, j}$. The nonlinear least-squares method is employed to minimize $F_{1}(\boldsymbol{\rho})^{2}+\ldots+F_{N}(\boldsymbol{\rho})^{2}$ with $\rho_{i, j}$ as variables. Updated normal vectors $\mathbf{N}_{i, j}$ are computed according to Eq. (14) using the optimized $\rho$ in the current iteration and the ray map. Iterations proceed to compute new $\rho$ until the 

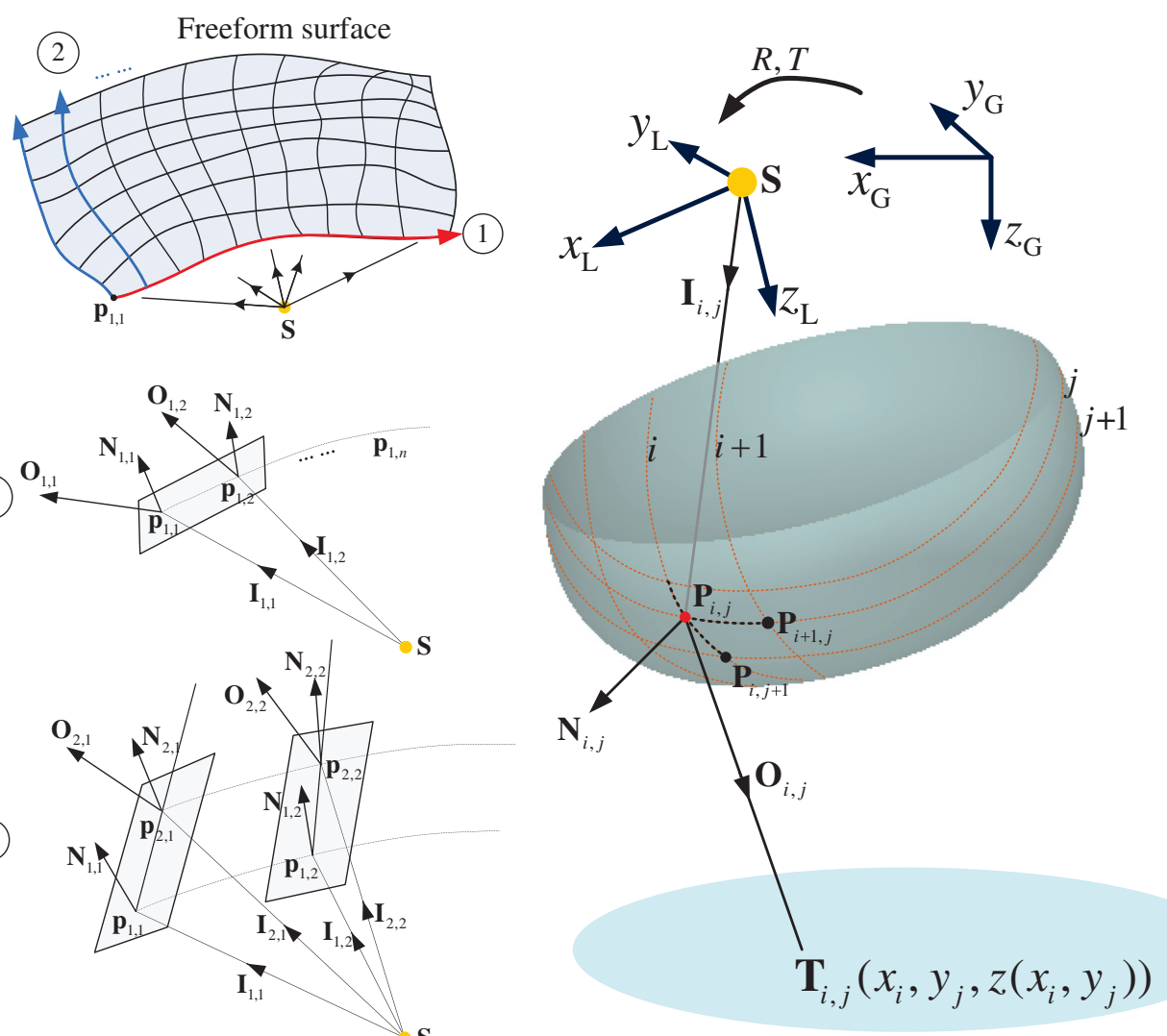

(a)

(b)

Fig. 7 Optical surface construction method. (a) Initial freeform surface construction and (b) optical surface optimization.

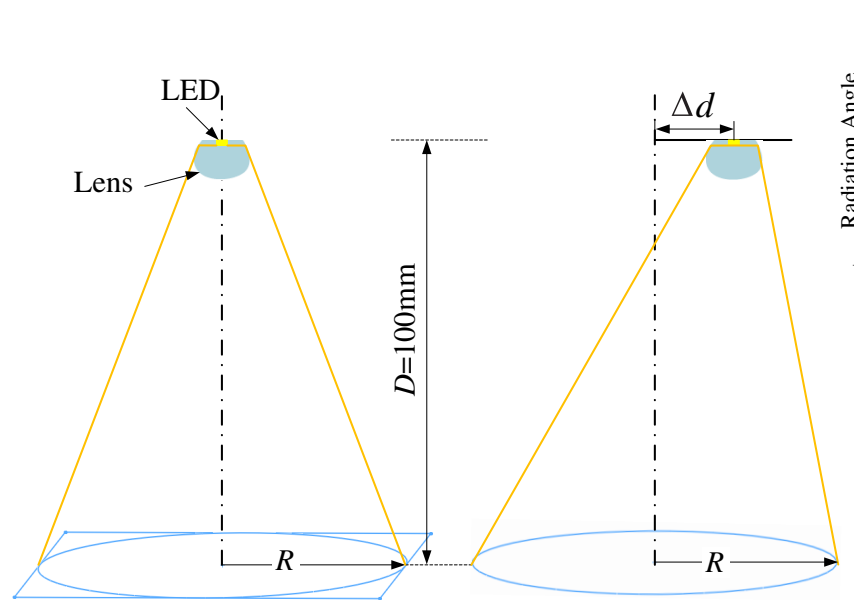

(a)

(b)

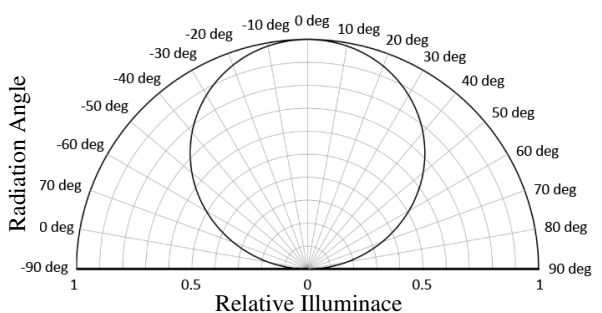

(c)

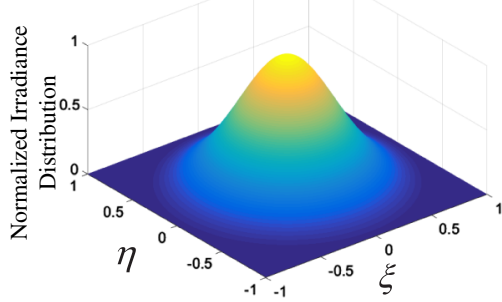

(d)

Fig. 8 Simulation setups for evaluating the freeform optical design method. (a) On-axis test: the axis of the LED coincides with the axis of target lighting regions, which are circular and square shapes in this test; (b) off-axis test: offsets $\Delta d=5 \mathrm{~mm}, 10 \mathrm{~mm}$, and $15 \mathrm{~mm}$ are between the axis of the LED and the axis of the target area. In this test, only circular target region is employed; (c) LED luminous intensity distribution from the LED's data sheet; ${ }^{42}$ and (d) the converted LED illuminance distribution using the method in Sec. 2.7. 
computed surface points satisfy the convergence condition $\left\|\boldsymbol{\rho}_{t}-\boldsymbol{\rho}_{t-1}\right\|<\delta$, where $t$ represents the current iteration number, and $\delta$ is the stopping threshold value. Finally, the optical surface can be represented using the freeform surface points with nonuniform rational basis spline. ${ }^{39}$

\subsubsection{Feedback modification}

Due to the zero-étendue source assumption, illuminance uniformity will be degraded using the LEDs with the extended sizes, especially in the case of designing small-volume optical lenses. This issue can be mitigated by employing a feedback modification method. ${ }^{40,41}$ Denote $E_{t}(x, y)$ as the desired illuminance distribution on a target region, $\tilde{E}_{t}(x, y)$ as the simulation

Table 2 Evaluation specifications of the freeform optical design method.

\begin{tabular}{lcc}
\hline LED & $1.7 \mathrm{~mm} \times 1.7 \mathrm{~mm} \times 0.3 \mathrm{~mm}, 118 \mathrm{Im}$ \\
\hline Lens & Material & Refractive index \\
& PMMA & 1.49 \\
\hline Target region & $\begin{array}{c}\text { Circular radius } \\
R=80 \mathrm{~mm}\end{array}$ & $\begin{array}{c}\text { Square side length } \\
2 R=160 \mathrm{~mm}\end{array}$ \\
\hline Source to target distance & & $100 \mathrm{~mm}$ \\
\hline Mesh dimensions & $81 \times 81, \xi \in[-1,1], \eta \in[-1,1]$, \\
& & \multicolumn{2}{c}{\begin{tabular}{c}
$\xi=0.025$ \\
\hline
\end{tabular}}
\end{tabular}

result of illuminance distribution by applying the freeform lenses. The modified desired illuminance distribution $E_{t}^{M}(x, y)$ for the next iteration can be defined as

$E_{t}^{M}(x, y)=\frac{E_{t}(x, y)}{\tilde{E}_{t}(x, y)} E_{t}(x, y)$.

As shown in the design framework in Fig. 3, illumination performance is evaluated in each iteration to check if a satisfiable illuminance uniformity is achieved. If yes, the freeform optical lens design is completed. Otherwise, another iteration will be executed to modify the surface of the freeform lenses.

\section{Results}

\subsection{Evaluation of Freeform Optical Lens Design Method}

In this section, we evaluate the performance of the freeform optical lens design method for the in vivo laparoscopic lighting system. Figures 8(a) and 8(b) show on-axis and off-axis tests, which were conducted separately using an optical design software package (TracePro, Lambda Research Corp.) to investigate the effectiveness of our optical design method in different application scenarios. We employed polymethyl methacrylate (PMMA) as the lens material with a refractive index of 1.49 , and Nichia NCSWE17A LEDs ${ }^{42}$ with a luminous flux of $118 \mathrm{~lm}$. To verify that our proposed method is flexible and capable of designing freeform optical lenses for illuminating
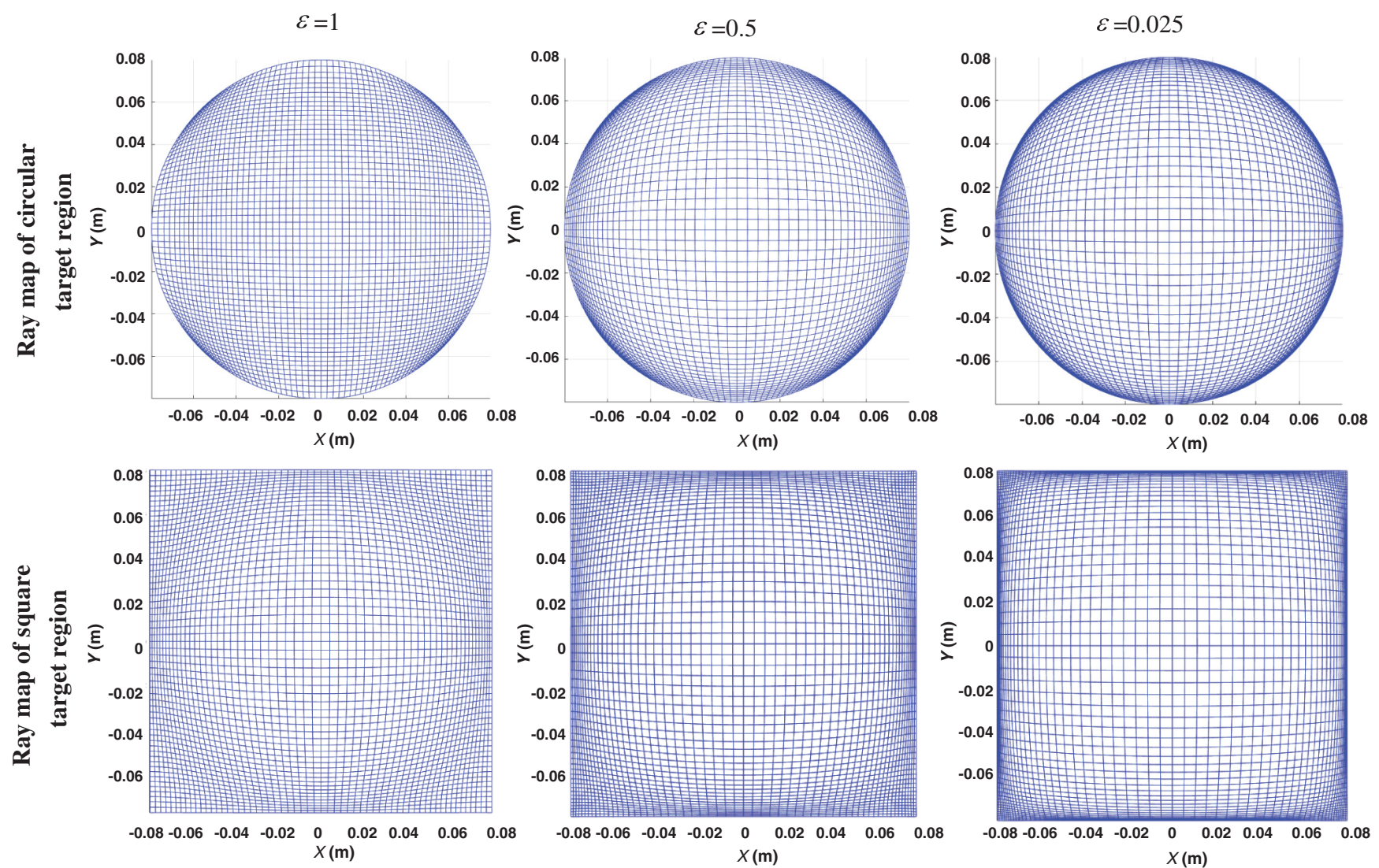

Fig. 9 On-axis ray maps of circular and square target regions computed with $\epsilon=1,0.5$, and 0.025 by using $81 \times 81$ mesh grids. For the purpose of clear visualization, we interpolate the meshes into $61 \times 61$ in this figure. 


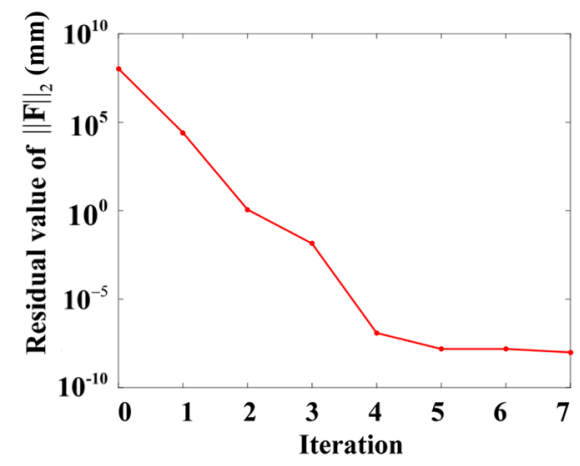

(a) Circular target region, $\varepsilon=1$

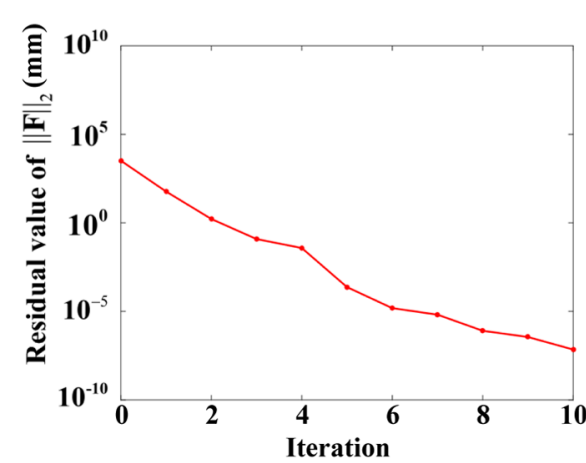

(d) Square target region, $\varepsilon=1$

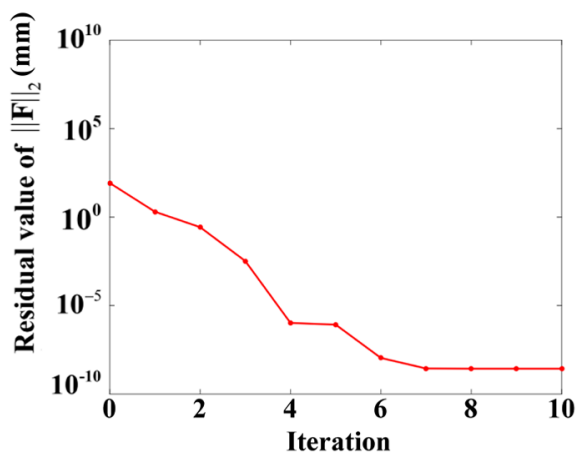

(b) Circular target region, $\varepsilon=0.5$

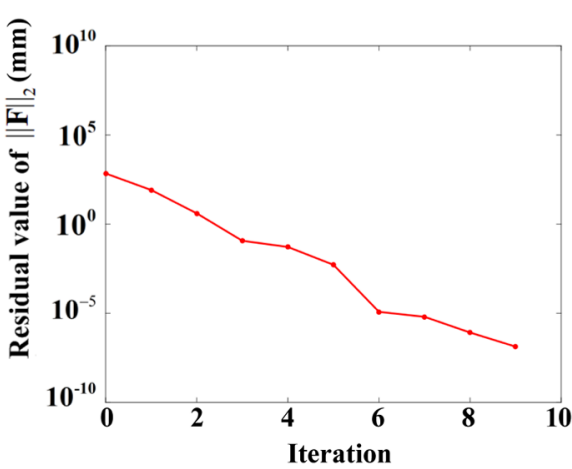

(e) Square target region, $\varepsilon=0.5$

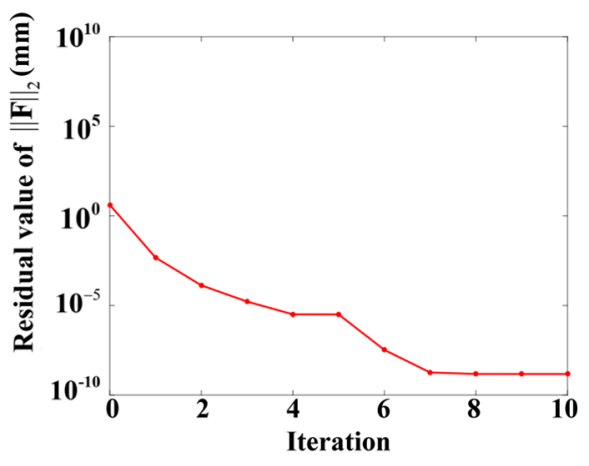

(c) Circular target region, $\varepsilon=0.025$

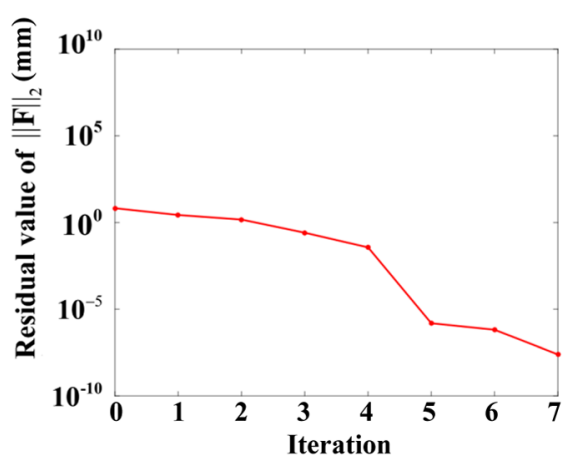

(f) Square target region, $\varepsilon=0.025$

Fig. 10 Convergent speeds of ray-mapping computations. (a)-(c) and (d)-(f) The convergent speeds in the cases of the circular area and the square area with $\epsilon=1,0.5$, and 0.025 , respectively.

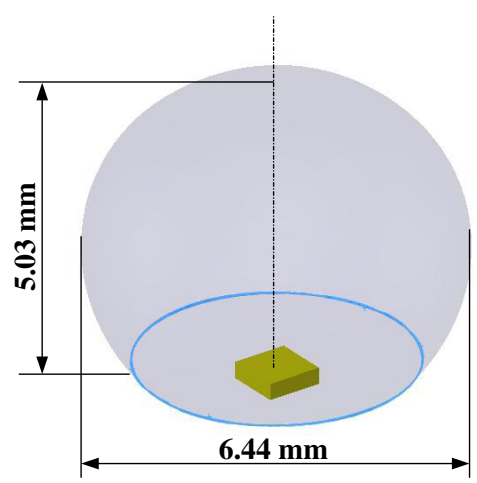

(a)

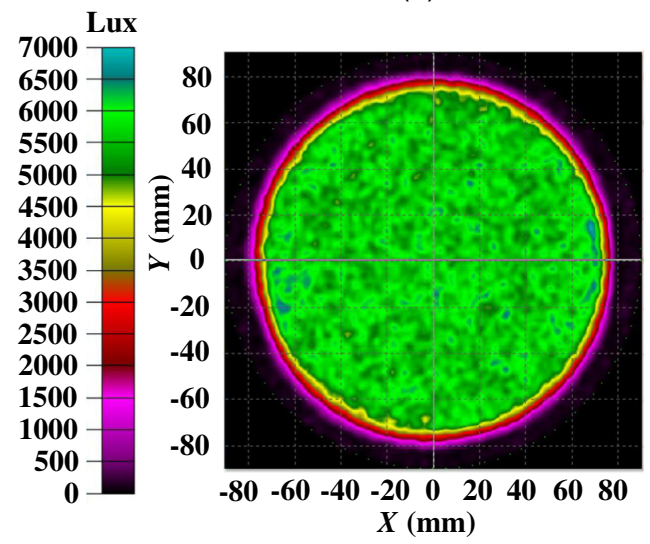

(c)

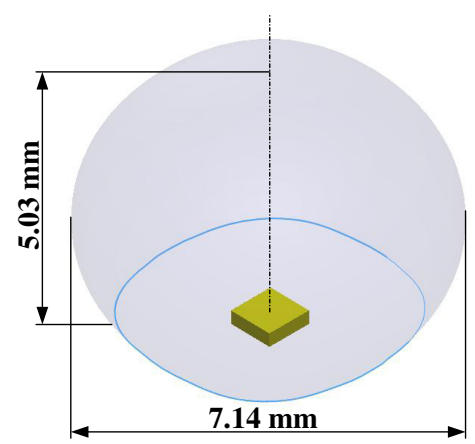

(b)
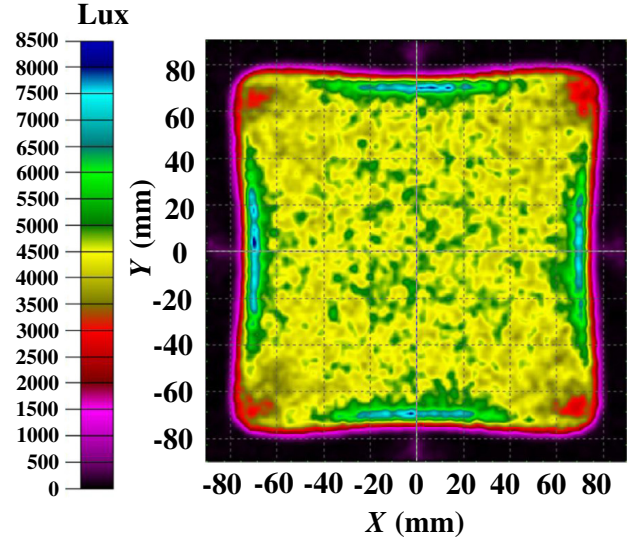

(d)

Fig. 11 On-axis freeform lens design for two different illumination patterns. (a) and (b) The profiles of the lenses for a circular area and a square area, respectively. (c) and (d) The illuminance uniformities performed by (a) and (b) separately on the target planes. 
Table 3 Optical performances of on-axis tests.

\begin{tabular}{lccc}
$\begin{array}{l}\text { Target } \\
\text { regions }\end{array}$ & $\begin{array}{c}\text { Optical } \\
\text { efficiency }\end{array}$ & $\begin{array}{c}\text { Average } \\
\text { illuminance }\end{array}$ & $\begin{array}{c}\text { Illuminance } \\
\text { uniformity }\end{array}$ \\
\hline Circular & $88.3 \%$ & $5,682.73 \mathrm{~lx}$ & $96.47 \%$ \\
Square & $91.2 \%$ & $4,648.19 \mathrm{~lx}$ & $94.23 \%$ \\
\hline
\end{tabular}

target areas with different patterns, we set the target area with a circular pattern and a square pattern for the on-axis illumination tests. The detailed specifications are summarized in Table 2.

\subsubsection{Ray map computation}

We first convert the luminous intensity distribution of the LED [Fig. 8(c)] to a normalized illuminance distribution [Fig. 8(d)].

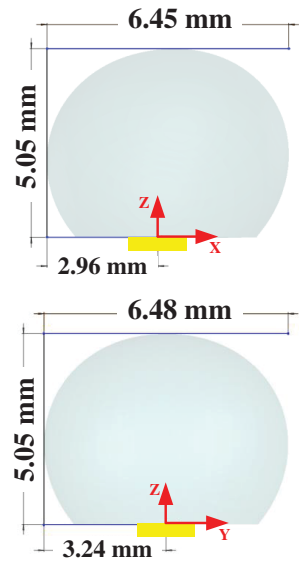

(a)

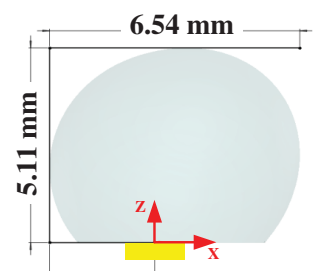

$2.74 \mathrm{~mm}$

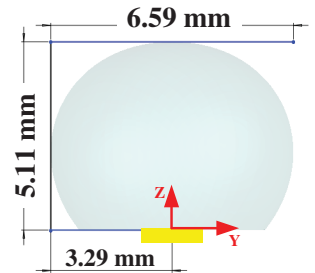

(d)
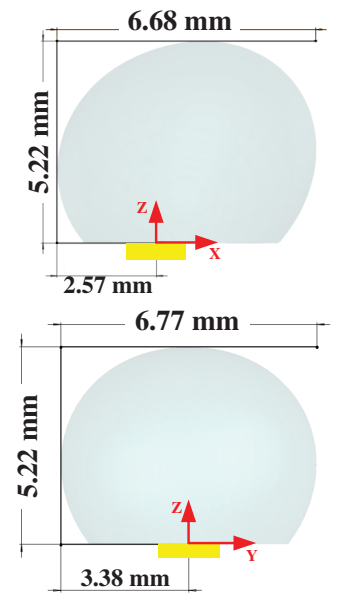

(g)

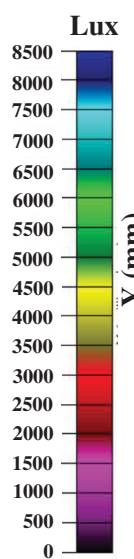

Lux

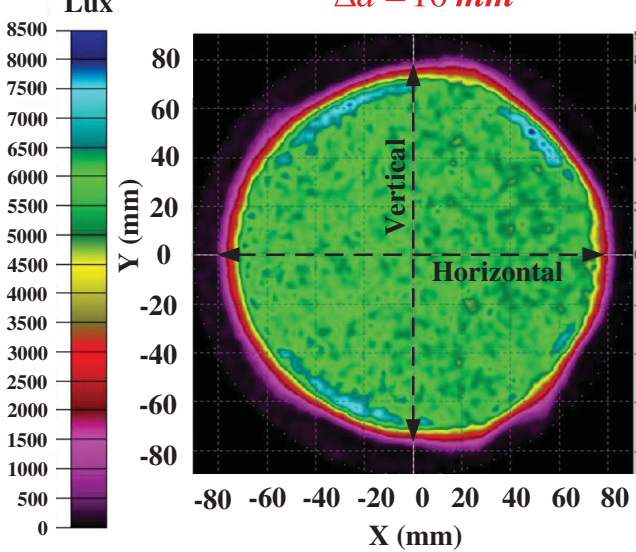

(e)

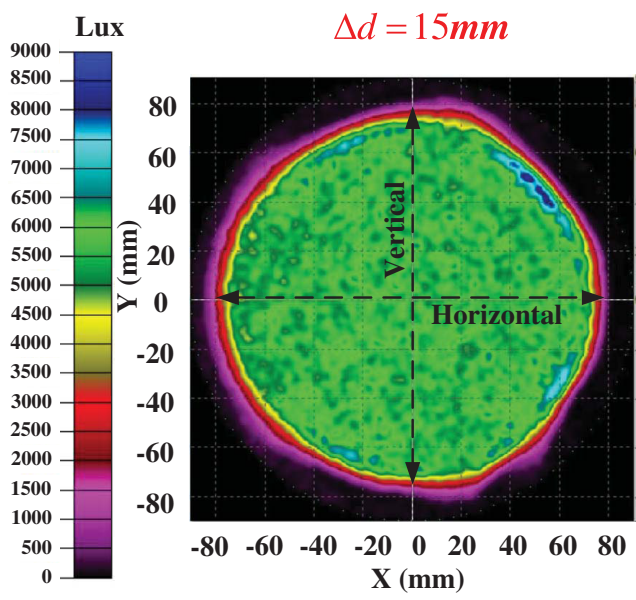

(h)

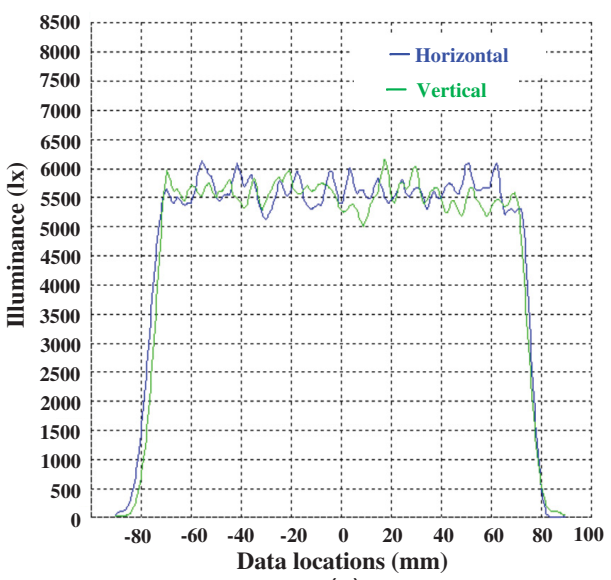

(c)

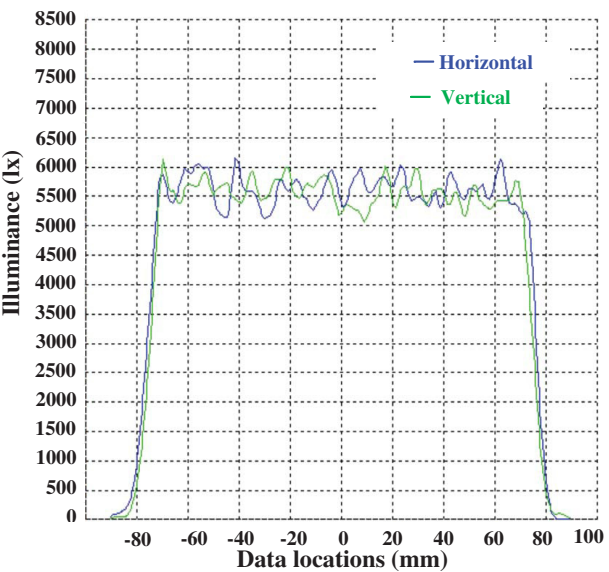

(f)

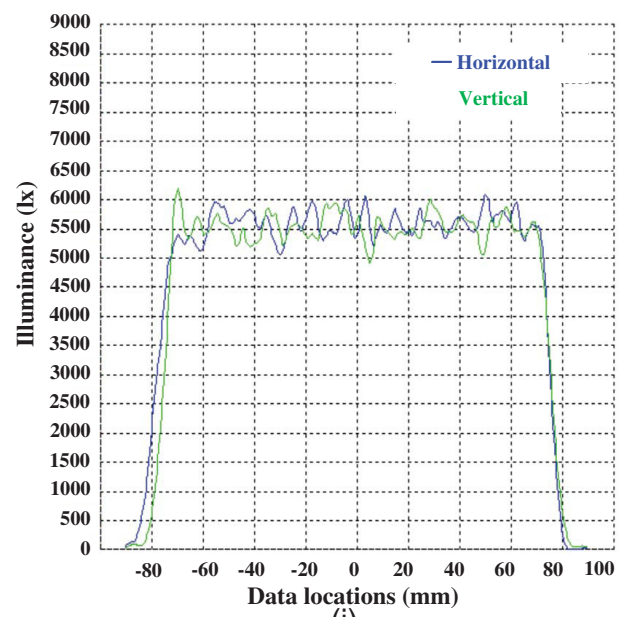

(i)

Fig. 12 Off-axis freeform lens design for a circular illumination area. (a)-(c) demonstrate of the designed freeform lens profile and illumination test results when the offset distance $\Delta d=5 \mathrm{~mm}$. Similarly, (d)-(f) and (g)-(i) demonstrate the lens design profiles and test results when $\Delta d=10 \mathrm{~mm}$ and $\Delta d=15 \mathrm{~mm}$ respectively. 
The computation domain of the $\operatorname{LED} \xi \in[-1,1], \eta \in[-1,1], \epsilon$ is then discretized by $81 \times 81$ mesh grids. The mesh size $h=0.025$ determines the minimum $\epsilon=0.025$ according to our ray-mapping algorithm. As shown in Fig. 9, we selected a sequence of $\epsilon$ as $1,0.5,0.025$ to approximate the numerical solution of the ray maps. For validating the effectiveness of the ray map generation method, we demonstrate the intermediate ray map results that are computed with $\epsilon=1,0.5,0.025$. The ray maps computed with $\epsilon=0.025$ are used to generate initial surfaces of freeform optical lenses for the LEDs.

Figure 10 shows the convergent speeds of our proposed ray map generation method. The convergent speeds are characterized by residual values of $\|\mathbf{F}\|_{2}$ in Eq. (11) and iteration times. The unit of residual value $\|\mathbf{F}\|_{2}$ of Eq. (11) is millimeter. Considering that the state-of-the-art highest manufacturing accuracy for a freeform optical lens is at the level of submicrometer $\left(10^{-4} \mathrm{~mm}\right)$, we conservatively set the convergence threshold at subnanometer $\left(10^{-7} \mathrm{~mm}\right)$. We observed that in all the tests, $\|\mathbf{F}\|_{2}$ can achieve the values of order $10^{-7}$ within 10 iterations.

\subsubsection{On-axis tests of freeform optical lens design}

Figure 8(a) shows the simulation setup of on-axis tests for the freeform optical lens design. A circular target region with a radius $R$ of $80 \mathrm{~mm}$ and a square target region with a side length $2 R$ of $160 \mathrm{~mm}$ are employed for the on-axis tests. The illumination distance from the LED to the target region center is set as $D=100 \mathrm{~mm}$. Figures $11(\mathrm{a})$ and 11(b) demonstrate the designed lens profiles with labeled dimensions. Figures 11(c) and $11(\mathrm{~d})$ show the simulated illuminance distributions on the target regions. The optical efficiencies of the freeform lenses are $88.3 \%$ and $90.5 \%$, respectively, with considering Fresnel losses. The illuminance uniformities can be quantified by

Uniformity $=\left(1-\frac{\sigma}{\mu}\right) \times 100$,

where $\sigma$ and $\mu$ are the standard deviation and mean of collected illuminance data, respectively. The optical performance of the on-axis tests is detailed in Table 3.

\subsubsection{Off-axis tests of freeform optical lens design}

The simulation setup of the off-axis tests is shown in Fig. 8(b). The illuminated region is set as a circular region with a radius $R$ of $80 \mathrm{~mm}$. The distance from the LED to the target plane is set as $D=100 \mathrm{~mm}$. Axis offsets $\Delta d=5 \mathrm{~mm}, 10 \mathrm{~mm}$, and $15 \mathrm{~mm}$ are introduced to evaluate the optical performance when the LED's axis and the target region's axis are not coincided. To construct freeform optical surfaces in this more generalized case, a transformation matrix is required to convert the ray map from the global coordinates to the LED's local coordinates. Figure 12 shows the designed lens profiles and the simulated illuminance results for each case. Due to the axis offsets, the optical lenses are no longer symmetric. So, we provide front and side views of the lenses, as shown in Figs. 12(a), 12(d), and 12(g). Figures 12(b), 12(e), and 12(h) show the simulated illuminance distributions on the circular target region. The optical efficiencies of the freeform lenses are $88.06 \%, 87.74 \%$, and $88.15 \%$, respectively with considering Fresnel losses. Figures 12(c), 12(f), and 12(i) show the illuminance uniformities along horizontal and vertical directions in the lighting regions. We summarize the optical performance of the off-axis tests in Table 4.
Table 4 Optical performance of off-axis tests.

\begin{tabular}{llcl}
\hline Offset $\Delta d$ & $\begin{array}{c}\text { Optical } \\
\text { efficiency }\end{array}$ & $\begin{array}{c}\text { Average } \\
\text { illuminance }\end{array}$ & $\begin{array}{c}\text { Illuminance } \\
\text { uniformity }\end{array}$ \\
\hline $5 \mathrm{~mm}$ & $88.06 \%$ & $5582.38 \mathrm{Ix}$ & Horizontal 95.48\% \\
& & & Vertical 95.19\% \\
$10 \mathrm{~mm}$ & $87.74 \%$ & $5503.14 \mathrm{Ix}$ & Horizontal 95.11\% \\
& & & Vertical 95.92\% \\
$15 \mathrm{~mm}$ & $88.15 \%$ & $5598.47 \mathrm{Ix}$ & Horizontal 95.63\% \\
& & & Vertical 95.79\% \\
\hline
\end{tabular}

\subsection{Integration and Evaluation of In Vivo Laparoscopic Lighting System}

Hitherto, we have verified the effectiveness of our proposed freeform optical lens design method. In the following, we demonstrate the evaluation of the in vivo laparoscopic lighting system to achieve desired lighting requirements indicated in Table 1.

\subsubsection{Final design of LEDs' freeform optical lenses}

Recall the configuration of the lighting system in Fig. 2. The lens installation position $L$ on the wings is set as $20.5 \mathrm{~mm}$. The open angle of the wings is set as $\beta=80 \mathrm{deg}$ for the extended mode. In the design, we set the lens volume with the maximum radial length $\rho_{\max }$ of $5.4 \mathrm{~mm}$ that guarantees the three lenses can fit in the robotic camera. The initial illumination distance $D$ is set as $100 \mathrm{~mm}$. The radius of the target circular area $R$ is set as $80 \mathrm{~mm}$. The specifications of the freeform optical lens design for the laparoscopic lighting system are summarized in Table 5.

Figure 13 shows the three-dimensional (3-D) design of the in vivo laparoscopic lighting system. Figure 13(a) shows the three views of the freeform lens. Figure 13(b) demonstrates the compactness of the lens, which satisfies the lens volume restriction. Figure 13(c) shows the integration of a lens and an LED in one wing. Figure 13(d) shows the 3-D structure of an assembled laparoscopic lighting system.

Table 5 Specifications of the lighting system setup.

\begin{tabular}{ll} 
Light sources & Nichia NCSWE17A, 118 Im \\
Lens material & PMMA, refractive index 1.49 \\
Lens maximum radial length $\left(\rho_{\max }\right)$ & $5.4 \mathrm{~mm}$ \\
Lens/LED position on a wing $(L)$ & $20.5 \mathrm{~mm}$ \\
Camera-to-target distance $(D)$ & $100 \mathrm{~mm}$ \\
Open angle of wings $(\beta)$ & $80^{\circ}$ \\
Radius of circular target region $(R)$ & $80 \mathrm{~mm}$ \\
\hline
\end{tabular}




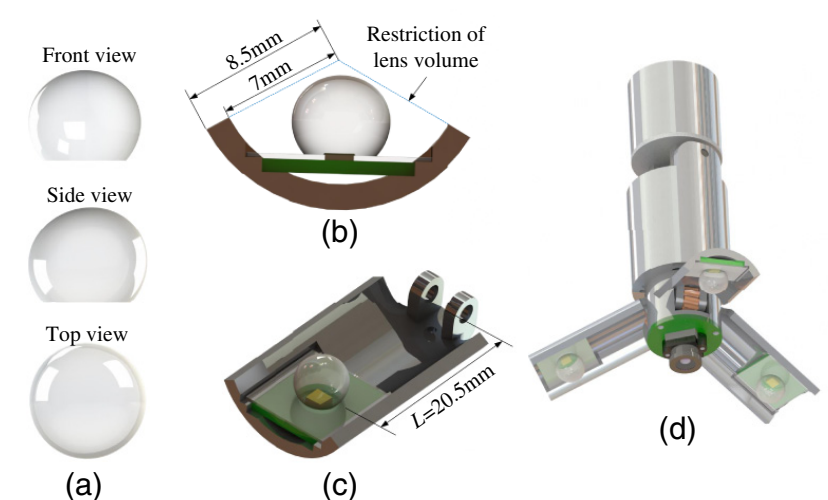

(a)

(c)

Fig. $133-D$ demonstration of the laparoscopic lighting system. (a) Three views of the lens; (b) demonstration of the lens volume and the restriction volume; (c) the LED and the lens installation on the wing; and (d) the assembled laparoscopic lighting system.

\subsubsection{Lighting performance on target region}

We evaluate the performance of the developed lighting system in accordance with the simulation setup in Table 5. Due to the symmetric arrangement of the three wings, a single LED is first energized, which emits light rays through its freeform lens. Figure 14(a) shows the illuminance distribution on the target region. Considering Fresnel losses, the optical efficiency of the designed freeform lens is $89.45 \%$, which means that for each LED $105.55 \mathrm{~lm}$ out of the total $118 \mathrm{~lm}$ luminous flux is successfully projected onto the desired target region. The average illuminance provided from the single LED is 5473.8 lx. By using the illuminance data shown in Fig. 14(b), the horizontal and vertical illuminance uniformities are computed as $95.87 \%$ and $94.78 \%$, respectively, by Eq. (19).

Figure 14(c) shows the illuminance distribution on the target region when all the LEDs are energized. In this case, the total luminous flux provided from the lighting system is $354 \mathrm{~lm}$, whereas the total luminous flux falling on the target region is $316.58 \mathrm{~lm}$. The optical efficiency is $89.43 \%$. The average illuminance on the target region is $12,441 \mathrm{~lx}$. Figure 14(d) shows that the illuminance uniformities along horizontal and vertical directions are $96.33 \%$ and $96.79 \%$, respectively. Figure 14(e) demonstrates the illuminance distribution on the target region with 3-D profile for better illustration. We summarize the evaluation results of the lighting performance in Table 6. It is obvious that the developed in vivo laparoscopic lighting system satisfies all of the design requirements imposed by Table 1 .

\subsubsection{Refocusing of light beams}

In MIS, after inserting the in vivo laparoscopic system inside the abdominal cavity, the distance $D$ between the camera and a target surgical area might be shorter than $100 \mathrm{~mm}$. Although the lighting system with the wings' angle at $\beta=80 \mathrm{deg}$ can still provide good illumination in that region, the illuminance uniformity will be degraded, and more energy will be wasted outside the camera's FOV.

Our proposed in vivo laparoscopic lighting system features a refocusing function, which is capable of controlling the light beams by adjusting the wings' angle to uniformly illuminate the target area within the camera's FOV when the camerato-target distance changes, as shown in Fig. 15(a). For instance,

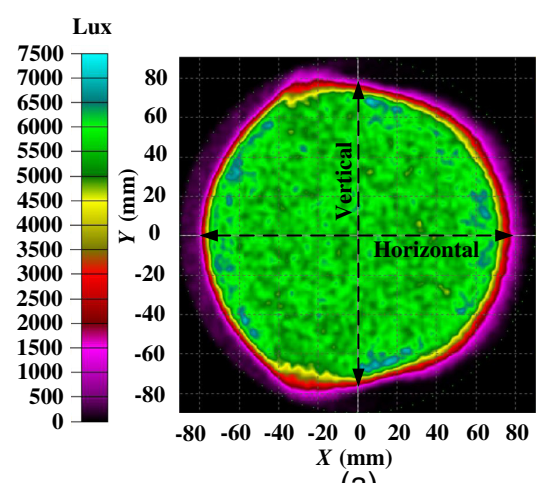

(a)

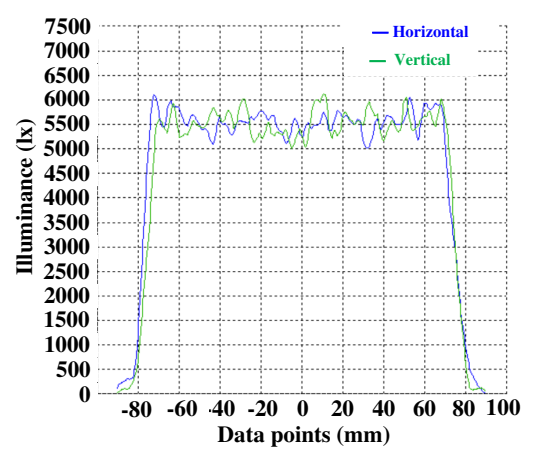

(b)

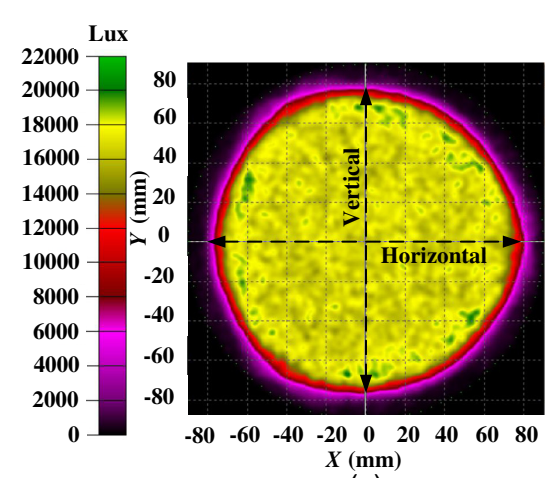

(c)

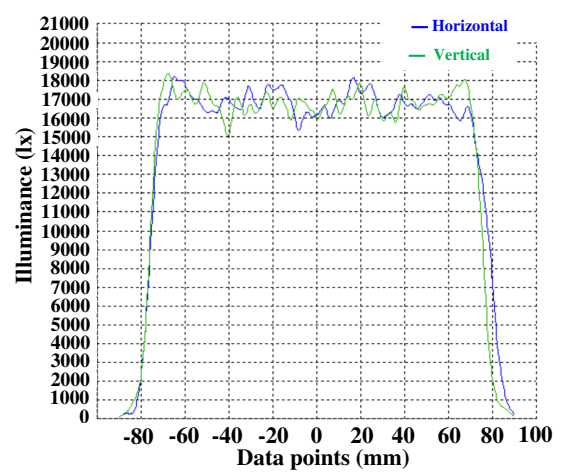

(d)

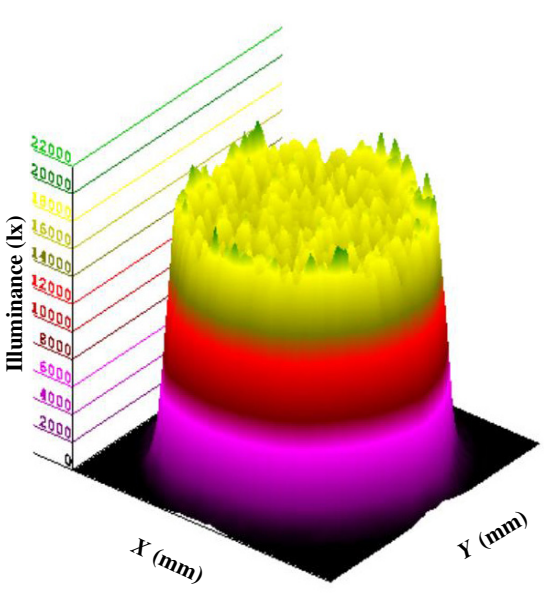

(e)

Fig. 14 Simulated illumination performance tests for the in vivo laparoscopic lighting system (circular area $R=80 \mathrm{~mm}, D=100 \mathrm{~mm}$ ). (a) The illuminance distribution with a single energized LED; (b) the illuminance uniformity of (a); (c) the illuminance distribution with three energized LEDs; (d) the illuminance uniformity of (c); and (e) the illuminance distribution of (c) in 3-D for better visualization. 
Table 6 Performance evaluation results of the lighting system.

\begin{tabular}{lccc}
$\begin{array}{l}\text { Number } \\
\text { of lighted } \\
\text { LEDs }\end{array}$ & $\begin{array}{c}\text { Optical } \\
\text { efficiency }\end{array}$ & $\begin{array}{c}\text { Average } \\
\text { illuminance }\end{array}$ & $\begin{array}{c}\text { Illuminance } \\
\text { uniformity }\end{array}$ \\
\hline 1 & $89.45 \%$ & $5473.8 \mathrm{Ix}$ & $\begin{array}{c}\text { Horizontal } 95.87 \% \\
\text { Vertical 95.78\% }\end{array}$ \\
3 & $89.43 \%$ & $16,981 \mathrm{~lx}$ & Horizontal 96.33\% \\
& & & Vertical 96.79\% \\
\hline
\end{tabular}

we set the desired target region with $D=60 \mathrm{~mm}$ (thick green line). When the wings' angle $\beta$ is set to be $80 \mathrm{deg}$, the illuminated region is covered by the yellow lines. This $\beta$ value works best for $D=100 \mathrm{~mm}$. To refocus the light on the target region when $D=60 \mathrm{~mm}$, we decrease the wings' opening angle from $\beta$ to $\beta-\Delta \beta$. We determine the value of $\Delta \beta$ using the included angle $\theta$ between the green dashed arrow and the yellow dashed arrow. According to the geometry of this setup, $\theta$ is calculated to be $6 \mathrm{deg}$. Similarly, to illuminate the target region with $D=80 \mathrm{~mm}$, the wings' angle should be decreased by $\theta=$ $3 \mathrm{deg}$ from the initial angle $\beta=80 \mathrm{deg}$.

Figures 15(b)-15(e) show the illuminance distributions by refocusing the light beams for the target planes at $D=60 \mathrm{~mm}$ and $D=80 \mathrm{~mm}$. In the case of Figs. 15(b) and 15 (c), $\beta$ is set at $74 \mathrm{deg}$. The average illuminance in the circular
Table 7 Lighting performance of the light refocusing tests.

\begin{tabular}{lll}
\hline & Case 1 & Case 2 \\
\hline Camera-to-target distance D & $60 \mathrm{~mm}$ & $80 \mathrm{~mm}$ \\
Radius of circular region R & $48 \mathrm{~mm}$ & $64 \mathrm{~mm}$ \\
Wings' angle $\beta$ & $74 \mathrm{deg}$ & $77 \mathrm{deg}$ \\
Optical efficiency & $92 \%$ & $90.9 \%$ \\
Average illuminance & $45,823 \mathrm{~lx}$ & $24,172 \mathrm{~lx}$
\end{tabular}

Illuminance uniformity

Horizontal $98.29 \%$ Horizontal $95.37 \%$

Vertical $98.22 \%$ Horizontal $95.98 \%$

region with a radius $R$ of $48 \mathrm{~mm}$ is calculated as 45,823 lx. The optical efficiency is about $92 \%$ with considering Fresnel losses. The illuminance uniformities along horizontal and vertical directions are $98.29 \%$ and $98.22 \%$. While in the case of Figs. 15(d) and 15(e), $\beta$ is set at $\beta=77 \mathrm{deg}$ to illuminate the target area with $D=80 \mathrm{~mm}$. The average illuminance in the circular region with a radius $R$ of $64 \mathrm{~mm}$ is calculated as $24,1721 x$. The optical efficiency is $90.9 \%$ with considering Fresnel losses. The horizontal and vertical illuminance uniformities are $95.37 \%$ and $95.98 \%$, respectively. The lighting performance of the refocused light beams is summarized in Table 7.

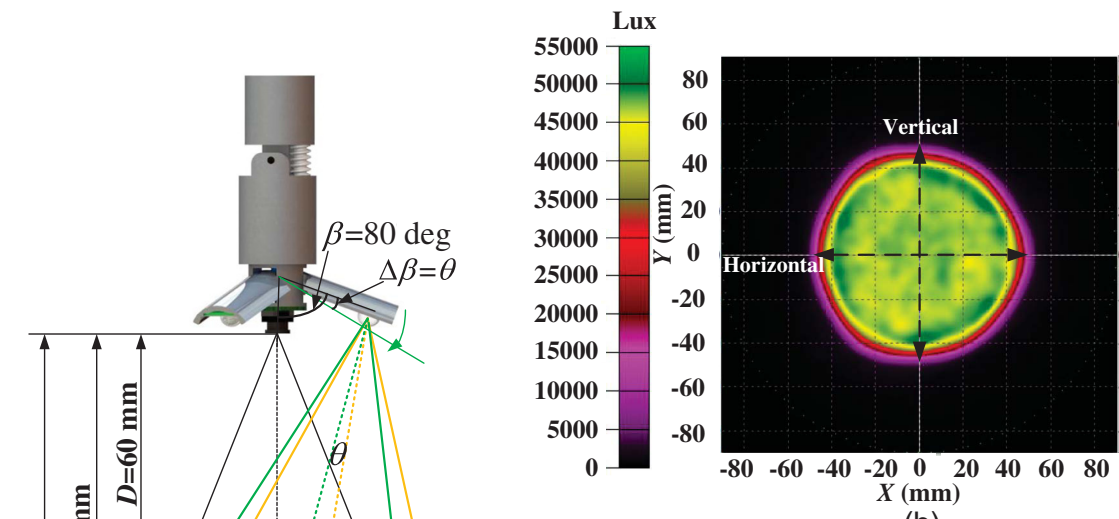

(b)

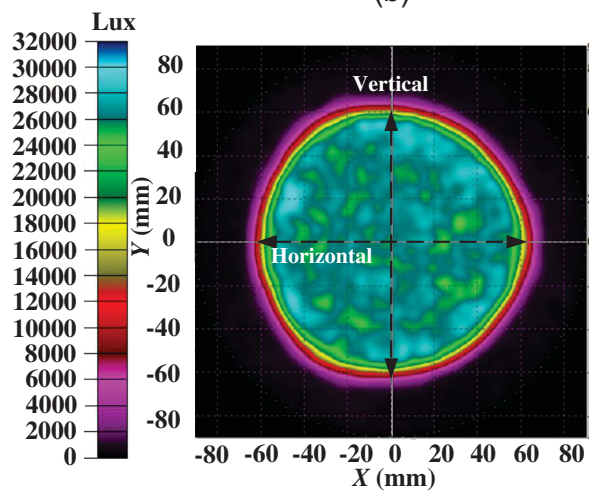

(d)

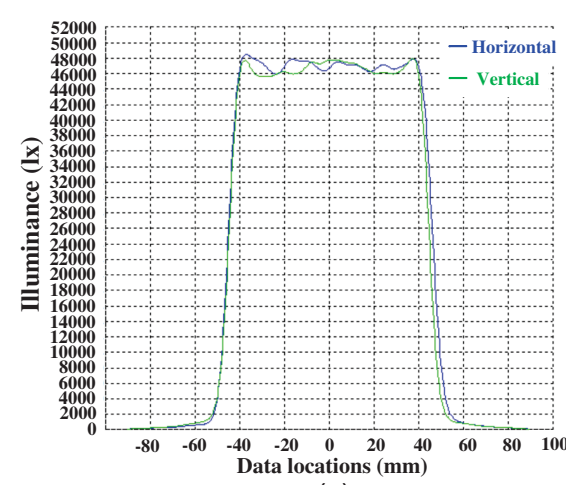

(c)

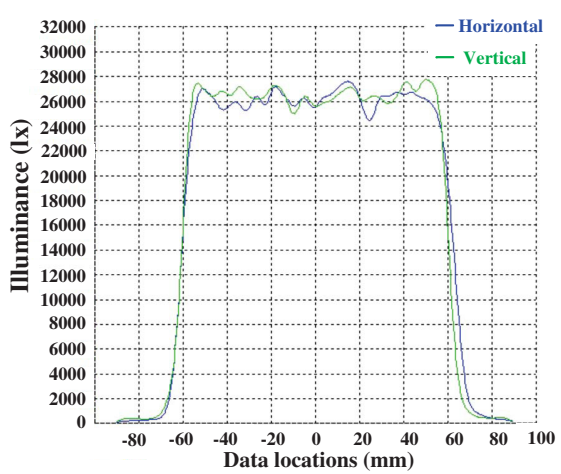

(e)

Fig. 15 Tests of refocusing light beams. (a) Demonstration of light beams refocusing on the regions with different distances by adjusting the wing angles $\beta$; (b) and (c) the illuminance distribution and the illuminance uniformity when $D=60 \mathrm{~mm}$; (d) and (e) the illuminance distribution and illuminance uniformity when $D=80 \mathrm{~mm}$. 


\section{Conclusion}

In this paper, we propose an innovative transformable in vivo laparoscopic lighting system design, which is able to carry well-designed freeform nonimaging optical lenses for providing high illuminance uniformity and high optical efficiency in a designated surgical area. Depending on the distance from the onboard camera to a surgical area, illuminated regions can be adjusted by changing the wings' opening angle without affecting the illuminance uniformity. To design freeform optical lenses, we present a ray-mapping-based method to construct freeform optical surface. A ray map that governed by a standard Monge-Ampere equation is efficiently computed by introducing a biharmonic operator in the PDE. An initial optical surface is constructed by Snell's law based on the generated ray map. To correct accumulated errors on the initial optical surface and improve the degraded illumination uniformity caused by the extended size of LEDs, we employ a surface optimization method and a feedback modification method. Simulation verifications validated that our proposed freeform optical lens design method features fast convergence speed of ray map generation $\left(\|\mathbf{F}\|_{2}\right.$ can achieve the value of $10^{-7}$ within 10 iterations), high illuminance uniformity (above $95 \%$ in average), and high optical efficiency with considering Fresnel losses (above 89\% in average).

\section{Disclosures}

The authors have no relevant financial interests in this article and no potential conflicts of interest to disclose.

\section{Acknowledgments}

Reza Yazdanpanah Abdolmalaki was supported by the National Science Foundation (ECCS-1309921). We would like to give special thanks to Professor Xiaobing Feng in the Department of Mathematics at the University of Tennessee, Knoxville, for discussing numerical solvers of Monge-Ampere equation.

\section{References}

1. T. Hu et al., "Insertable surgical imaging device with pan, tilt, zoom, and lighting," Int. J. Rob. Res. 28(10), 1373-1386 (2009).

2. C. A. Castro et al., "A wireless robot for networked laparoscopy," IEEE Trans. Biomed. Eng. 60(4), 930-936 (2013).

3. S. Platt, J. Hawks, and M. Rentschler, "Vision and task assistance using modular wireless in vivo surgical robots," IEEE Trans. Biomed. Eng. 56(6), 1700-1710 (2009).

4. B. S. Terry et al., "An integrated port camera and display system for laparoscopy," IEEE Trans. Biomed. Eng. 57(5), 1191-1197 (2010).

5. J. Cadeddu et al., "Novel magnetically guided intra-abdominal camera to facilitate laparoendoscopic single-site surgery: initial human experience," Surg. Endoscopy 23(8), 1894-1899 (2009).

6. M. Simi et al., "Magnetically activated stereoscopic vision system for laparoendoscopic single-site surgery," IEEE/ASME Trans. Mechatronics 18(3), 1140-1151 (2013).

7. X. Liu, G. J. Mancini, and J. Tan, "Design of a unified active locomotion mechanism for a capsule-shaped laparoscopic camera system," in IEEE Int. Conf. on Robotics and Automation (ICRA '14), pp. 2449-2456 (2014).

8. X. Liu et al., "Design of a magnetic actuated fully insertable robotic camera system for single-incision laparoscopic surgery," IEEE/ASME Trans. Mechatronics 21(4), 1966-1976 (2016).

9. P. Swain et al., "Development and testing of a tethered, independent camera for notes and single-site laparoscopic procedures," Surg. Endoscopy 24(8), 2013-2021 (2010).

10. "Stryker endoscopic cameras," http://www.stryker.com/en-us/products/ Endoscopy/VisualizationandDocumentationSystems/EndoscopicCameras/ index.htm (23 March 2017).
11. "Olympus 4K cameras," http://medical.olympusamerica.com/products/ VISERA-4K-UHD-System (23 March 2017).

12. R. Wu, Y. Qin, and H. Hua, "Improved illumination system of laparoscopes using an aspherical lens array," Biomed. Opt. Express 7(6), 2237-2248 (2016).

13. "Laparoscopic trocars," http://www.laparoscopic.md/instruments/trocar (23 March 2017).

14. G. B. Hanna, A. B. Cresswell, and A. Cuschieri, "Shadow depth cues and endoscopic task performance," Arch. Surg. 137(10), 1166-1169 (2002).

15. A. C. Lee et al., "Solid-state semiconductors are better alternatives to arc-lamps for efficient and uniform illumination in minimal access surgery," Surg. Endoscopy 23(3), 518-526 (2009).

16. H. Ries and J. Muschaweck, "Tailored freeform optical surfaces," J. Opt. Soc. Am. A 19(3), 590-595 (2002).

17. J. S. Schruben, "Formulation of a reflector-design problem for a lighting fixture," J. Opt. Soc. Am. 62(12), 1498-1501 (1972).

18. M. G. Crandall and P. L. Lions, "Viscosity solutions of Hamilton-Jacobi equations," Trans. Am. Math. Soc. 277(1), 1-45 (1983).

19. X. Feng and M. Neilan, "Vanishing moment method and moment solutions for fully nonlinear second order partial differential equations," J. Sci. Comput. 38(1), 74-98 (2009).

20. L. Wang, K. Qian, and Y. Luo, "Discontinuous free-form lens design for prescribed irradiance," Appl. Opt. 46(18), 3716-3723 (2007).

21. "FLEXIPATH Trocars, FP020," http://www.ethicon.com/healthcareprofessionals/products/access/trocars/other (23 March 2017).

22. "OV7850 CMOS sensor," http://www.ovt.com/download/sensorpdf/ 208/OmniVision_OV7850.pdf (23 March 2017).

23. "Sunex imaging lens," http://www.optics-online.com/OOL/DSL/ DSL871.PDF (23 March 2017).

24. “All facts for choosing LED optics correctly," http://ledil.fi/sites/default/ files/Documents/Technical/Articles/Article_1.pdf (23 March 2017).

25. K. Brix, Y. Hafizogullari, and A. Platen, "Designing illumination lenses and mirrors by the numerical solution of Monge-Ampère equations," J. Opt. Soc. Am. A 32(11), 2227-2236 (2015).

26. R. Wu et al., "Freeform illumination design: a nonlinear boundary problem for the elliptic Monge-Ampére equation," Opt. Lett. 38(2), 229-231 (2013).

27. D. Michaelis, P. Schreiber, and A. Bräuer, "Cartesian oval representation of freeform optics in illumination systems," Opt. Lett. 36(6), 918920 (2011).

28. F. R. Fournier, W. J. Cassarly, and J. P. Rolland, "Fast freeform reflector generation usingsource-target maps," Opt. Express 18(5), 5295-5304 (2010).

29. V. Oliker, "Mathematical aspects of design of beam shaping surfaces in geometrical optics," in Trends in Nonlinear Analysis, M. Kirkilionis et al., Ed., pp. 193-224, Springer, Berlin, Heidelberg (2003).

30. A. Bäuerle et al., "Algorithm for irradiance tailoring using multiple freeform optical surfaces," Opt. Express 20(13), 14477-14485 (2012).

31. M. M. Sulman, J. Williams, and R. D. Russell, "An efficient approach for the numerical solution of the Monge-Ampère equation," Appl. Numer. Math. 61(3), 298-307 (2011).

32. Z. Feng, B. D. Froese, and R. Liang, "Freeform illumination optics construction following an optimal transport map," Appl. Opt. 55(16), 43014306 (2016).

33. B. D. Froese, "A numerical method for the elliptic Monge-Ampère equation with transport boundary conditions," SIAM J. Sci. Comput. 34(3), A1432-A1459 (2012).

34. R. Wu et al., "Initial design with $\mathrm{L}^{2}$ Monge-Kantorovich theory for the Monge-Ampère equation method in freeform surface illumination design," Opt. Express 22(13), 16161-16177 (2014).

35. Y. Brenier, "Polar factorization and monotone rearrangement of vectorvalued functions," Commun. Pure Appl. Math. 44(4), 375-417 (1991).

36. X. Feng and M. Neilan, "Mixed finite element methods for the fully nonlinear Monge-Ampère equation based on the vanishing moment method," SIAM J. Numer. Anal. 47(2), 1226-1250 (2009).

37. L. Lapidus and G. F. Pinder, Numerical Solution of Partial Differential Equations in Science and Engineering, John Wiley \& Sons, Hoboken, New Jersey (2011).

38. R. Swaminathan, S. K. Nayar, and M. D. Grossberg, "Framework for designing catadioptric projection and imaging systems," in Proc. of the 
IEEE Int. Workshop on Projector Camera Systems, IEEE Computer Society Press, Los Alamitos, California (2003).

39. L. Piegl and W. Tiller, The NURBS Book, 2nd ed., Springer Science \& Business Media, New York (1997).

40. Y. Luo et al., "Design of compact and smooth free-form optical system with uniform illuminance for led source," Opt. Express 18, 9055-9063 (2010).

41. R. Wester et al., "Designing optical free-form surfaces for extended sources," Opt. Express 22, A552-A560 (2014).

42. "Nichia NCSWE17A," http://www.nichia.co.jp/en/product/led_product_ data.html?type $=\% 27$ NCSWE17A\%27 (23 March 2017).

Xiaolong Liu is a research assistant professor at the University of Tennessee, Knoxville, Tennessee, USA. He received his BS and MS degrees in electrical and computer engineering from Northeastern University, China, in 2008 and 2010, respectively, and his $\mathrm{PhD}$ in biomedical engineering from the University of Tennessee, Knoxville, Tennessee, USA, in 2015. His current research interests include surgical robotics, biomedical optics, and other surgical-related engineering designs.

Reza Yazdanpanah Abdolmalaki received his BS degree in mechanical engineering from Amirkabir University of Technology,
Tehran, Iran, in 2011 and his MS degree from the University of Tehran, Iran, 2014. Currently, he is pursuing his $\mathrm{PhD}$ in the Department of Mechanical, Biomedical and Aerospace Engineering (MABE) at the University of Tennessee, Knoxville, Tennessee, USA. His current research interests include surgical robotics, control systems, robotics, and engineering design.

Gregory J. Mancini received his MD degree in general surgery from Mercer University School of Medicine in Macon, Georgia, in 2000. Currently, he is an associate professor of surgery at the University of Tennessee, Knoxville. His clinical practice and academic efforts focus on the area minimally invasive surgery. $\mathrm{He}$ is board certified in general surgery by the American Board of Surgery and is a fellow of the American College of Surgeons.

Jindong Tan received his $\mathrm{PhD}$ in electrical and computer engineering from Michigan State University, East Lansing, Michigan, USA, in 2002. Currently, he is a professor and associate department head with the Department of Mechanical, Aerospace and Biomedical Engineering, The University of Tennessee, Knoxville, Tennessee, USA. His current research interests include mobile sensor networks, augmented reality and biomedical imaging, dietary assessment, and mobile manipulation. 